\title{
Ligninolytic activity of the Penicillium chrysogenum and Pleurotus ostreatus fungi involved in the biotransformation of synthetic multi-walled carbon nanotubes modify its toxicity
}

\author{
Gladys Juárez-Cisneros ${ }^{1}$, Jesús Campos-García ${ }^{\text {Corresp., }}{ }^{2}$, Sharel Pamela Díaz-Pérez ${ }^{2}$, Javier Lara-Romero ${ }^{3}$, Dhirendra \\ Kumar Tiwari ${ }^{4}$, Juan Manuel Sánchez-Yáñez ${ }^{1}$, Homero Reyes-De la Cruz ${ }^{5}$, Sergio Jiménez-Sandoval ${ }^{6}$, Javier \\ Villegas ${ }^{\text {Corresp. } 1}$ \\ 1 Laboratorio de Interacción Suelo Planta Microorganismo, Instituto de Investigaciones Químico Biológicas, Universidad Michoacana de San Nicolás de \\ Hidalgo, Morelia, Michoacán, Mexico \\ 2 Laboratorio de Biotecnología Microbiana, Instituto de Investigaciones Químico Biológicas, Universidad Michoacana de San Nicolás de Hidalgo, Morelia, \\ Michoacán, Mexico \\ 3 Facultad de Ingeniería Química, Universidad Michoacana de San Nicolás de Hidalgo, Morelia, Michoacán, Mexico \\ 4 El Colegio de Michoacán, Ladipa, La Piedad, La Piedad, Michoacán, Mexico \\ 5 Laboratorio de Control Traduccional, Instituto de Investigaciones Químico Biológicas, Universidad Michoacana de San Nicolás de Hidalgo, Morelia, \\ Michoacán, Mexico \\ 6 Unidad Querétaro, Centro de Investigación y de Estudios Avanzados del IPN, Querétaro, Querétaro, Mexico \\ Corresponding Authors: Jesús Campos-García, Javier Villegas \\ Email address: jesus-campos@umich.mx,vilj4455@yahoo.com.mx
}

Multi-walled carbon nanotubes (MWCNTs) are of multidisciplinary scientific interest due to their exceptional physicochemical properties and a broad range of applications. However, they are considered potentially toxic nanoparticles when they accumulate in the environment. Given their ability to oxidize resistant polymers, mycorremediation with lignocellulolytic fungi are suggested as biological alternatives to the mineralization of MWCNTs. Hence, this study involves the ability of two fungi specie to MWCNTs biotransformation by laccase and peroxidases induction and evaluation in vivo of its toxicity using Caenorhabditis elegans worms as a model. Results showed that the fungi Penicillium chrysogenum and Pleurotus ostreatus were capable to grow on media with MWCNTs supplemented with glucose or lignin. Activities of lignin-peroxidase, manganeseperoxidase, and laccase in cultures of both fungi were induced by MWCNTs. Raman, FTIR spectroscopy, HR-TEM, and TGA analyses of the residue from the cultures of both fungi revealed structural modifications on the surface of MWCNTs and its amount diminished, correlating the MWCNTs structural modifications with the laccase-peroxidase activities in the fungal cultures. Results indicate that the degree of toxicity of MWCNTs on the $C$. elegans model was enhanced by the structure modification associated with the fungal ligninolytic activity. The toxic effect of MWCNTs on the in vivo model of worms reveals the increment of reactive oxygen species as a mechanism of toxicity. Findings indicate that the MWCNTs can be subject in nature to biotransformation processes such as the fungal 
metabolism, which contribute to modify their toxicity properties on susceptible organisms and contributing to environmental elimination. 
1 Ligninolytic activity of the Penicillium chrysogenum and Pleurotus ostreatus

2 fungi involved in the biotransformation of synthetic multi-walled carbon

3 nanotubes modify its toxicity

4 Gladys Juárez-Cisneros ${ }^{1}$, Jesús Campos-García ${ }^{2 *}$, Sharel Díaz-Pérez ${ }^{2}$, Javier Lara-Romero ${ }^{3}$,

5 Dhirendra K. Tiwari ${ }^{4}$, Juan Manuel Sánchez-Yáñez ${ }^{1}$, Homero Reyes-De la Cruz ${ }^{2}$, Sergio

6 Jiménez-Sandoval $^{5}$, Javier Villegas ${ }^{1 *}$.

7

$8 \quad{ }^{1}$ Laboratorio de Interacción Suelo Planta Microorganismo, Instituto de Investigaciones Químico

9 Biológicas, Universidad Michoacana de San Nicolás de Hidalgo, Morelia, Michoacán, México.

$10{ }^{2}$ Laboratorio de Biotecnología Microbiana, Instituto de Investigaciones Químico ${ }^{3}$ Biológicas,

11 Universidad Michoacana de San Nicolás de Hidalgo, Morelia, Michoacán, México.

$12{ }^{3}$ Facultad de Ingeniería Química, Universidad Michoacana de San Nicolás de Hidalgo, Morelia, 13 Michoacán, México.

$14{ }^{4}$ El Colegio de Michoacán, Ladipa, La Piedad, Michoacán, México

$15{ }^{5}$ Laboratorio de Control Traduccional, Instituto de Investigaciones Químico Biológicas,

16 Universidad Michoacana de San Nicolás de Hidalgo, Morelia, Michoacán, México.

\section{*Corresponding Authors:}

\section{Jesús Campos-García}

Instituto de Investigaciones Químico Biológicas, Universidad Michoacana de San Nicolás de Hidalgo, Ciudad Universitaria, Morelia, Michoacán, 58030, México.

Email address: jesus.campos@umich.mx

Javier Villegas

Instituto de Investigaciones Químico Biológicas, Universidad Michoacana de San Nicolás de

\section{Abstract}


40

41

42

43

44

45

46

47

48

49

50

51

52

53

54

55

56

57

58

59

60

61

62

63

64

65

66

67

68

69

70

71

72

73

74

75

76

77

78

79

Multi-walled carbon nanotubes (MWCNTs) are of multidisciplinary scientific interest due to their exceptional physicochemical properties and a broad range of applications. However, they are considered potentially toxic nanoparticles when they accumulate in the environment. Given their ability to oxidize resistant polymers, mycorremediation with lignocellulolytic fungi are suggested as biological alternatives to the mineralization of MWCNTs. Hence, this study involves the ability of two fungi specie to MWCNTs biotransformation by laccase and peroxidases induction and evaluation in vivo of its toxicity using Caenorhabditis elegans worms as a model. Results showed that the fungi Penicillium chrysogenum and Pleurotus ostreatus were capable to grow on media with MWCNTs supplemented with glucose or lignin. Activities of lignin-peroxidase, manganese-peroxidase, and laccase in cultures of both fungi were induced by MWCNTs. Raman, FTIR spectroscopy, HR-TEM, and TGA analyses of the residue from the cultures of both fungi revealed structural modifications on the surface of MWCNTs and its amount diminished, correlating the MWCNTs structural modifications with the laccaseperoxidase activities in the fungal cultures. Results indicate that the degree of toxicity of MWCNTs on the C. elegans model was enhanced by the structure modification associated with the fungal ligninolytic activity. The toxic effect of MWCNTs on the in vivo model of worms reveals the increment of reactive oxygen species as a mechanism of toxicity. Findings indicate that the MWCNTs can be subject in nature to biotransformation processes such as the fungal metabolism, which contribute to modify their toxicity properties on susceptible organisms and contributing to environmental elimination.

\section{Introduction}

Carbon nanotubes (CNTs) are nanomaterials that consist of one or several sheets of crystalline carbon rolled in concentric cylinders (Saifuddin et al. 2013). They are of scientific, commercial, and environmental interest due to their unique mechanical, electrical, and optical properties (Zaytseva 2016). Specifically, CNTs are increasingly used in areas such as biomedical, genetics, electronics, cosmetics, pharmacology, and agriculture (Burlaka et al. 2015; De Volder et al. 2013). However, given the high stability of CNTs, they can accumulate in the ecosystem and lead to environmental and health risks (Shin et al. 2015). Reports suggest that CNTs nanoparticles by their diverse chemical structures, shape, size, and low solubility are potentially toxic for diverse organisms such as microorganisms (Ge et al. 2016; Shin et al. 2015; Uo et al. 2011; Yadav et al. 2016), plants (Rong et al. 2018; Lin et al. 2009; Tan et al. 2009), and animals (Bhattacharya et al. 2013; Petersen et al. 2011). In this context, recent studies focused on determining biological alternatives to degrade or mineralize CNTs to avoid their possible health risks that have been described (Bhattacharya et al. 2013; Petersen et al. 2008).

In the last decade, in vitro studies report that oxidative enzymes from human, microbial, or plant can catalyze CNT degradation (Modugno et al. 2016). Additionally, peroxidase isolated from Armoracia rusticana in presence of $\mathrm{H}_{2} \mathrm{O}_{2}$ or $\mathrm{FeCl}_{3}$ partially degrades CNTs structures of a single thick layer or single-walled carbon nanotubes (SWCNTs) (Allen et al. 2009; Magrez et al. 2006)

Peer] reviewing PDF | (2020:11:55553:2:0:NEW 19 Feb 2021) 
80

81

82

83

84

85

86

87

88

89

90

91

92

93

94

95

96

97 98

99

100

101

102

103

104

105

106

107

108

109

110

111

112

113

114

115

116

117

118

119

and multi-walled carbon nanotubes (MWCNTs) (Petersen et al. 2008; Zhao et al. 2012). Similarly, reports indicate that SWCNTs are susceptible to degradation by enzymes from animal cells such as myeloperoxidase (Kagan et al. 2010; Vlasova et al. 2011), lactoperoxidase (Bhattacharya et al. 2013), and eosinophil-peroxidase (Andõn et al. 2013). Research suggest that mycorremediation by basidiomycetes and deuteromycetes represents a biological alternative to alleviate the accumulation of carbon nanomaterials such as MWCNTs through extracellular peroxidases, such as manganese-peroxidase (MnP), lignin-peroxidase (LiP), or laccase, which exhibit the potential to oxidize MWCNTs (Fujii et al. 2013; Janusz et al. 2013); through unspecific enzymes that catalyze the oxidation of compounds such as polycyclic aromatic hydrocarbons, phenols, organophosphates, and pesticides (Gan et al. 2009; Haritash and Kaushik 2009; Ting et al. 2011). Phenol-oxidases such as laccase catalyzes the oxidation, decarboxylation or methoxylation of a wide spectrum of phenolic compounds, aromatic amines, and polynucleoaromatic hydrocarbons. Whereas, LiP are relatively nonspecific enzymes for substrates, these can oxidize aromatic compounds with high redox potential such as veratryl alcohol, methoxybenzenes, and nonphenolic groups of the lignin; contrary to, the $\mathrm{MnP}$ are specific by substrates (Qu et al. 2013). In this context, compounds such as pyrene, benzo (a) pyrene, acenaphthene, phenanthrene, anthracene, and fluorantrene can be oxidized by enzymes such as LiP, MnP, and laccases (Barr and Aust, 1994; Wang et al. 2009).

On the other hand, it has been documented that white rot fungi through an enzymatic mechanism by laccases and $\mathrm{MnP}$ excreted by $P$. chrysosporiume transformed and oxidized pristine MWCNT by modifying functional groups of the carbon structure $(\mathrm{C}=\mathrm{O}$ and $\mathrm{O}-\mathrm{H}$ radicals), leading to shortening of MWCNTs structure (Barr and Aust, 1994; Ma et al. 2019). Enzymes from Sparassis latifolia can be utilized to biotransform SWCNTs into compounds with lower toxicity and used for mineralization of carbon nanomaterials (Chandrasekaran et al. 2014), also as peroxidases and laccases from Trametes versicolor and Phlebia tremellosa that partially degrades SWCNTs (Zhang et al. 2014). Thus, fungal enzymes are suggested as candidates to biotransform or biodegrade carbon nanomaterials into simpler and susceptible compounds and represent a biological alternative to control the persistence of nanoparticles in the environment (Ge et al. 2016; Parks et al. 2015). However, to date, there is a paucity of studies that establish the roles played by microbial communities in the biotransformation of MWCNTs. This is a key point because fungi are the main recyclers of organic material in nature. Caenorhabditis elegans is a saprophytic nematode that lives in the liquid phase of the soil and leaf-litter environments, it has been widely utilized as an in vivo animal model in toxicology to evaluate the compounds toxicity such as carbon-based nanomaterials among others (Chen et al. 2013). Therefore, the objective of the present study involved an in vitro evaluation of the capacity of two fungi, Pleurotus ostreatus and Penicillium chrysogenum to biotransformation/biodegradation of synthetic MWCNTs, thereby also evaluating its toxicity in C. elegans worms as in vivo soilbased model. 
120

121

122

123

124

125

126

127

128

129

130

131

132

133

134

135

136

137

138

139

140

141

142

143

144

145

146

147

148

149

150

151

152

153

154

155

156

157

158

159

\section{Materials \& Methods}

\section{MWCNT specifications}

The MWCNTs used in the study exhibit the following characteristics: Outer diameter of 6-13 $\mathrm{nm}$, internal diameter of 2.0-4.0 $\mathrm{nm} \times 2.5-20 \mu \mathrm{m}$ length, average wall thickness $7-13$ graphene layers, and purity $>98 \%$ (Aldrich, Cat 698849).

\section{Culture conditions}

In the study, Pleurotus ostreatus and Penicillum chrysogenum were provided from the collection of microorganisms in our laboratory (microscopic morphology is showed in Figure S1, supplementary material). The initial inoculum was prepared by culturing each fungus in a potato dextrose agar medium (PDA; BD-Bioxon) for an incubation period of $7 \mathrm{~d}$ at $22^{\circ} \mathrm{C}$.

Subsequently, discs of the agar plate corresponding to approximately $1 \mathrm{~cm}^{2}$ for each fungus were inoculated in a 100-mL liquid medium with the following composition (g/L): $\mathrm{NH}_{4} \mathrm{NO}_{3} 5.0$, $\mathrm{CuSO}_{4} 0.01, \mathrm{MgSO}_{4} 1.5, \mathrm{KH}_{2} \mathrm{PO}_{4} 1.5, \mathrm{~K}_{2} \mathrm{HPO}_{4} 1.5, \mathrm{NaCl} 0.9$, yeast extract 1.0, bromothymol blue $10 \mathrm{ppm}$, and $1 \mathrm{~mL} / \mathrm{L}$ of trace elements solution prepared as follows $(\mathrm{g} / \mathrm{L}): \mathrm{H}_{3} \mathrm{BO}_{3} 2.86$, $\mathrm{ZnSO}_{4}-7 \mathrm{H}_{2} \mathrm{O} 0.22, \mathrm{MnCl}_{2}-7 \mathrm{H}_{2} \mathrm{O}$ 1.81, and $\mathrm{KMnO}_{4}$ 0.09. The carbon source used corresponded to $0.5 \mathrm{~g} / \mathrm{L}$ glucose and/or $2 \mathrm{~g} / \mathrm{L}$ of residual lignin from semi-purified wheat straw based on Baltierra-Trejo (2016) and $10 \mu \mathrm{g} / \mathrm{mL}$ of MWCNTs at a $\mathrm{pH}$ of 5.5. The fungi cultures were incubated on a rotary shaker at $150 \mathrm{rpm}$ for $28 \mathrm{~d}$ at $30{ }^{\circ} \mathrm{C}$.

\section{Determination of enzymatic activities}

Specifically, 10-mL samples were collected at intervals corresponding to 7 days from the culture media of $P$. chrysogenum and $P$. ostreatus fungi and enzymatic activities were determined in supernatants. Supernatants were obtained by centrifugation at $8000 \times \mathrm{g}$ (Thermo Fisher SL 40R) for $15 \mathrm{~min}$ at $4{ }^{\circ} \mathrm{C}$ and, thereby eliminating mycelium and residual lignin. The lignin-peroxidase activity was determined via the oxidation of veratryl alcohol to veratraldehyde by measuring absorbance at $310 \mathrm{~nm}$ using a spectrophotometer (BioTek Instrument Inc-Epoch), the molar extinction coefficient $(\varepsilon) 9300 \mathrm{M}^{-1} \mathrm{~cm}^{-1}$. The reaction mixture was prepared with $400 \mu \mathrm{L}$ of supernatant on $2.5 \mathrm{~mL}$ of sodium acetate buffer, $100 \mathrm{mM}, \mathrm{pH} 3.0,1.0 \mathrm{~mL}$ of veratryl alcohol (Sigma) $10 \mathrm{mM}$, and $100 \mu \mathrm{L}$ of $\mathrm{H}_{2} \mathrm{O}_{2} 10 \mathrm{mM}$. The manganese-peroxidase activity was determined by the oxidation of $\mathrm{MnSO}_{4}$ to tartrate by measuring absorbance at $240 \mathrm{~nm}$ using a spectrophotometer, $\varepsilon 6500 \mathrm{M}^{-1} \mathrm{~cm}^{-1}$ (Gao et al. 2011). The reaction mixture was prepared with $400 \mu \mathrm{L}$ of supernatant, $3.4 \mathrm{~mL}$ of sodium malonate buffer (Sigma) $50 \mathrm{mM}, \mathrm{pH} 4.5,0.1 \mathrm{~mL}$ $\mathrm{MnSO}_{4} 15 \mathrm{mM}, 100 \mu \mathrm{L} \mathrm{H}_{2} \mathrm{O}_{2} 10 \mathrm{mM}$. Laccase activity was determined via the oxidation of 2,2'acino-bis-(3-ethyl-benzothiazoline-6-sulfonic acid) (ABTS) by measuring absorbance at $420 \mathrm{~nm}$ using a spectrophotometer; $\varepsilon$ of oxidized ABTS was $36,500 \mathrm{M}^{-1} \mathrm{~cm}^{-1}$. The reaction mixture was prepared with $300 \mu \mathrm{L}$ of supernatant, $2.4 \mathrm{~mL}$ of sodium acetate buffer $25 \mathrm{mM}$, pH 3.0, and 300 $\mu \mathrm{L}$ of ABTS $10 \mathrm{mM}$ (Ibrahim et al. 2011; Palmieri et al. 1997). 
160

161

162

163

164

165

166

167

168

169

170

171

172

173

174

175

176

177

178

179

180

181

182

183

184

185

186

187

188

189

190

191

192

193

194

195

196

197

198

199

\section{Analyses of MWCNTs}

The content and chemical modifications of MWCNTs was analyzed at the beginning ( 0 days) and end (28 days) of the assay in the fungi cultures ( $5-\mathrm{mL}$ by sample), which samples were dried using an oven at $72{ }^{\circ} \mathrm{C}$ for $48 \mathrm{~h}$ and later incinerated at $700{ }^{\circ} \mathrm{C}$ for $5 \mathrm{~h}$ to mineralize noncrystalline organic matter. Subsequently, the ashes obtained were pulverized and suspended in distilled water or used directly for further qualitative and quantitative analyses.

The samples ( $0.5 \mathrm{mg}$ of dry sample) analyzed by Raman spectroscopy were dispersed in $50 \mu \mathrm{L}$ water, aliquots of $10 \mu \mathrm{L}$ were placed on slides, five drops for each sample, allowed to dry at room temperature to be analyzed in Raman spectrometer (Lab Ram HR Evolution, Horiba) with a spectral resolution of $5 \mathrm{~cm}^{-1}$ using a $20-\mathrm{mW}$ He-Ne laser at $632.8 \mathrm{~nm}$ visual excitation, and the spectral range was scanned from $110 \mathrm{~cm}^{-1}$ to $3690 \mathrm{~cm}^{-1}$ with an integration time of $5 \mathrm{~s}$ (LaraRomero et al. 2017).

Attenuated total reflection Fourier transform infrared (ATR-FTIR) spectroscopy of samples obtained under the aforementioned conditions were analyzed, and the spectra were recorded via a Nicolet iS10 spectrophotometer (Thermo Scientific) by the attenuated total reflection (ATR) technique.

Thermogravimetric (TGA) analysis was carried out using a microbalance (SDT Q600-TA Instruments), where $50 \mathrm{mg}$ of dry sample was exposed to air-heated with temperatures of 10 to $700{ }^{\circ} \mathrm{C}$ with intervals of $5^{\circ} \mathrm{C} / \mathrm{min}$, to obtain TGA combustion curve.

High-resolution transmission electron microscopy (HR-TEM) was performed by using a Philips CM-200 analytical TEM operating at $200 \mathrm{kV}$. Specimens for HR-TEM analysis were prepared by dispersing the samples in acetone via sonication for $2 \mathrm{~min}$ and air-drying a drop of the suspension on a perforated carbon-coated $\mathrm{Cu}^{\circ}$ grid at room temperature (Lara-Romero et al. 2017).

\section{Caenorhabditis elegans killing assays}

In the study, C. elegans Bristol N2 and CL2166 dvIs19[pAF15(Pgst-4::GFP::NLS)] worms (provided by the Caenorhabditis Genetics Center, University of Minnesota). Worms clone (CL2166) that contains the GFP reporter protein fusion into the transgene dvIs19 [(pAF15)gst4p::GFP::NLS] III, oxidative stress-inducible GFP, whose reporter driven by the promoter of the glutathione S-transferase gene (gst-4p). In C. elegans, expression of gst-4 is activated by redox cycling compounds, electrophiles, and heavy metals (Leung et al.). Worms were synchronized by hypochlorite isolation of eggs from gravid adults, and the eggs were then hatched in a S-basal medium. Specifically, L1 larvae were transferred onto nematode growth medium (NGM) (Stiernagle, 2006) plates seeded with Escherichia coli JM101 strain, which was previously grown on the plates as a food source, and incubated at $20{ }^{\circ} \mathrm{C}$ for $4-5 \mathrm{~d}$ until the larvae reached the young-adult phase. Worms were rinsed from the plates and washed in an S-basal medium. In each experiment, 20 worms were dispensed into each well of 36-well plates, and this was followed by incubation with $1-\mathrm{mL}$ cultures media or residues obtained from incineration at 300 
200

201

202

203

204

205

206

207

208

209

210

211

212

213

214

215

216

217

218

219

220

221

222

223

224

225

226

227

228

229

230

231

232

233

234

235

236

237

238

239

${ }^{\circ} \mathrm{C}$ for $4 \mathrm{~h}$. The plates were incubated at $18{ }^{\circ} \mathrm{C}$, and live worms were scored at 12 -h intervals. A worm was considered as dead when it no longer responded (moved) to a touch stimulus. Two independent assays by duplicate were conducted for each worm group.

\section{Microscopy}

For superoxide $\left(\mathrm{O}_{2}{ }^{*-}\right)$ determination, worms samples were incubated with $5 \mu \mathrm{g} / \mathrm{mL}$ dihydroethidium (DHE, Molecular Probes, Invitrogen) at $30^{\circ} \mathrm{C}$ for $30 \mathrm{~min}$ in the dark, washed with phosphates buffer and observed by using a fluorescent and phase contrast inverted microscope (Nikon TE300) with PlanFluor 4X, 10X dry lenses and an AmScope ML300 3.1 MP digital color camera. From the fluorescence microscopy images-obtained, the same number of nematodes from each treatment was utilized to determinate fluorescence intensity using Image J Software (NIH). Data represent the mean value \pm standard error (SE) of three independent assays with $\mathrm{n}=10$ for each treatment. One-way ANOVA analysis was carried out, with Tukey's post-hoc test. Values for $P<0.05$ are shown with different lower-case letters.

\section{Results}

\section{Fungi growth and induction of ligninolytic enzymes in cultures containing MWCNTs} Assimilation of compounds such as lignin has been widely documented and correlated with the synthesis of enzymes such as LiP, MnP, or laccase (Baltierra-Trejo et al. 2016; Chen et al. 2011; Ting et al. 2011). Thus, we determined the MWCNTs biodegradation capacity of $P$. chrysogenum and $P$. ostreatus fungi in cultures media supplemented with carbon nanomaterials, thereby inducing ligninolytic enzymes via the addition of lignin as a carbon source with or without glucose supplementation. Results showed that both the $P$. chrysogenum and $P$. ostreatus fungi were capable to grow on media with lignin or glucose as the sole carbon source and supplemented with MWCNTs $(10 \mu \mathrm{g} / \mathrm{mL})$ (Figure 1).

Additionally, the results showed that in the P. ostreatus culture medium, lignin used as the carbon source induced the activities for laccase, $\mathrm{LiP}$, and $\mathrm{MnP}$ comparing with glucose wherein it did not induce enzymatic activities at similar levels (Figure 2). It should be noted that the activity levels of the enzymes increased when MWCNTs were supplemented with lignin in the culture medium and even when MWCNTs were used without addition of carbon source (Figure 2A-C). Similar results were obtained in the culture media of $P$. chrysogenum fungus (Figure 2DF). Also, laccase activity exceeds $\mathrm{LiP}$ and $\mathrm{MnP}$ activities in cultures of $P$. chrysogenum, and the presence of MWCNTs increased MnP activity at similar levels than that induced with lignin (Figure 2).

Chemical modifications into MWCNTs by growth of $P$. ostreatus and $P$. chrysogenum fungi Raman and infrared spectroscopy also as thermogravimetric analyses were conducted to perform an in-depth study of the effect of ligninolytic enzymes in cultures of both $P$. ostreatus and $P$. 
240 chrysogenum strains over possible chemical modifications into MWCNTs nanomaterial. The

241 Raman spectra indicated that the addition of MWCNTs in the P. ostreatus cultures led to

242 significant changes in the bands ID $\left(\sim 1370 \mathrm{~cm}^{-1}\right)$, IG $\left(\sim 1600 \mathrm{~cm}^{-1}\right)$, and 2D $\left(\sim 2640 \mathrm{~cm}^{-1}\right)$

243 (Figure 3A-D). However, the presence of MWCNTs + lignin and also MWCNTs + lignin +

244 glucose in both the $P$. ostreatus and $P$. chrysogenum cultures clearly modified the ID, IG, and 2D

245 bands of the Raman spectra, and they were observed as broad signals with the apparition of

246 additional bands in the range of $1350-1550 \mathrm{~cm}^{-1}$ (Figure 3B-C and 3F-G). Conversely, the

247 addition of MWCNTs alone or with glucose in the culture medium where the $P$. chrysogenum

248 fungus was grown did not exhibit significant changes in the ID, IG, and 2D bands of the Raman

249 spectra thereby indicating the integral presence MWCNTs (Figure 3E and 3H), similar to

250 MWCNTs without fungi exposition (Figure 3I).

251 With respect to the Raman spectra, it is observed that in the MWCNTs containing samples from

252 the fungi cultures, the IG band changed in terms of intensity and definition of peaks and

253 exhibited a group of peaks within the range of $1500-1600 \mathrm{~cm}^{-1}$ in a manner independent of the

254 carbon source (lignin or glucose). The IG band deformation was significantly observed in the

255 spectra of samples containing MWCNTs + lignin when compared with that in the glucose

256 supplementation.

257 The presence of lignin in the cultures supplemented with MWCNTs and the correlation with

258 increases in the enzymatic activities indicated the total loss of the $2 \mathrm{D}$ band at $\sim 2640 \mathrm{~cm}^{-1}$ in the

259 Raman spectra (Figure 3A-C and 3F-G).

260 Additionally, the cultures of $P$. ostreatus and $P$. chrysogenum in the presence of lignin plus

261 MWCNTs after 28 days of incubation were analyzed using FTIR spectrometry. We observed that

262 the material obtained at beginning of the assay showed characteristic signals at $\sim 3260 \mathrm{~cm}^{-1}$ and

$263 \sim 1700 \mathrm{~cm}^{-1}$ that correspond to $\mathrm{O}-\mathrm{H}$ functional groups (Figure 4, black and light blue lines).

264 These radicals are characteristic of disordered regions of MWCNTs, which conforms the

265 nucleation sites for hydrogen atoms. Other signals attributed to stretch vibrations of C-H at

$266 \sim 2200 \mathrm{~cm}^{-1}$ and $\mathrm{C}-\mathrm{H}_{\mathrm{X}}$ groups of the disordered sp3 region and vibration rings at $600 \mathrm{~cm}^{-1}$ were

267 characteristics of MWCNTs (Al-Rekaby 2018; Lehman et al. 2011). Conversely, we observed

268 that the FTIR spectra of the culture residue from $P$. ostreatus cultivated with MWCNTs + lignin

269 indicated the absence of the characteristic bands of the MWCNTs, also a characteristic peak of

270 the $\mathrm{COOH}$ functional radicals was identified at $\sim 1700 \mathrm{~cm}^{-1}$ (Figure 4). The FTIR spectrum of the

271 culture residue from $P$. chrysogenum grown on medium with MWCNTs + lignin indicated the

272 lower intensity of the characteristic peak of the $\mathrm{O}-\mathrm{H}$ groups at $\sim 3260 \mathrm{~cm}^{-1}$ and absence of peaks

273 characteristic of MWCNTs. Contrariwise, the presence of $\mathrm{COOH}$ signal at $\sim 1700 \mathrm{~cm}^{-1}$ and $\mathrm{CO}$

274 groups at $\sim 1100 \mathrm{~cm}^{-1}$ were identified (Figure 4).

275

276 Structural modifications into the MWCNTs by growth of $\boldsymbol{P}$. ostreatus and $\boldsymbol{P}$. chrysogenum

277 fungi

278 Chemical changes in the MWCNTs observed in Raman and IR spectroscopy analyses were

279 examined by high resolution transmission electronic microscopy (HR-TEM). It was observed 
280 that the structure of the MWCNTs was heterogeneous after the growth of $P$. chrysogenum and $P$.

281

282

283

284

285

286

287

288

289

290

291

292

293

294

295

296

297

298

299

300

301

302

303

304

305

306

307

308

309

310

311

312

313

314

315

316

317

318

319

ostreatus fungi in media supplemented with MWCNTs. The HR-TEM images showed a clear decrease in the amount of MWCNTs aggregates (Figure 5A-J). The intensity of structural changes in the MWCNTs were dependent on the fungus strain and the treatment that was applied. The structural modifications were more evident in the cultures of $P$. ostreatus using MWCNTs + lignin as carbon sources (Figure 5A-E). It should be noted that structural changes in the surface of the nanotubes were observed and included loss of layers and changes in both the internal and external diameters of the MWCNTs structures (Figure 5N-O). The average internal and external diameters of the synthetic MWCNTs that exhibit homogeneous structures were around $4 \mathrm{~nm}$ and $20 \mathrm{~nm}$, respectively (Figure 5K-M); while the diameters of MWCNTs from the samples analyzed after the cultivation for 28 days of the $P$. ostreatus fungus in the medium supplemented with MWCNTs + lignin were approximately $8 \mathrm{~nm}$ and $16 \mathrm{~nm}$, respectively (Figure $5 \mathrm{~N}-\mathrm{O})$.

In addition, thermogravimetric analysis (TGA) of dried samples from the cultures of the $P$. ostreatus and P. chrysogenum fungi showed that the MWCNTs amount clearly decreased after 28 days of growth in the medium supplemented with lignin or glucose (Figure 6). Compounds that are burned below $300-480{ }^{\circ} \mathrm{C}$ correspond to lignin/glucose and cellular biomass; while that compounds that remains unburned above $>500-600{ }^{\circ} \mathrm{C}$ suggest to be crystalline carbon nanomaterials such as MWCNTs and graphene. In the TGA analysis, the MWCNTs amount in the $\sim 1.0 \mathrm{mg}$ of each samples at the beginning of the experiment was submitted to incineration around $500-700{ }^{\circ} \mathrm{C}$, the determination of sample weight were reduced in the treatments with $P$. ostreatus after 28 days of incubation. Interestingly, in the treatment with $P$. ostreatus grown on lignin + glucose + MWCTNs, the major weight loss in sample was observed, suggesting that $P$. ostreatus degraded MWCNTs significantly more efficient than P. chrysogenum (Figure 6).

\section{Toxic effects of MWCNTs in C. elegans worms}

With respect to the possible toxic effects of the MWCNTs over the live organisms, we determined their toxicity by using supernatants from cultures of fungi containing MWCNTs on the toxicological animal model of $C$. elegans wild type strain and the recombinant worms clone (CL2166) that contains the GFP reporter protein fusion, oxidative stress-inducible (Leung et al. 2013).

Worms were placed in the presence of cultures media filtered and culture media incinerated at $300^{\circ} \mathrm{C}$ and worm survival was determined over time as described in Material and Methods. The culture medium containing MWCNTs exhibited $\sim 80 \%$ survival of nematodes versus $\sim 95 \%$ with medium alone at $72 \mathrm{~h}$ of worms exposure; interestingly, the addition of glucose, lignin, or both in the medium strongly increased the culture medium toxicity, showing $\sim 70-80 \%$ worms survival (Figure 7). On the other hand, the samples from cultures of both $P$. ostreatus and $P$. chrysogenum that were submitted to incineration to avoid effect of biological-origin compounds present in the culture media after fungi growth, exhibited different levels of survival on the nematodes, corresponding between $45-60 \%$ when they were incubated by $72 \mathrm{~h}$ with a medium

Peer] reviewing PDF | (2020:11:55553:2:0:NEW 19 Feb 2021) 
320

321

322

323

324

325

326

327

328

329

330

331

332

333

334

335

336

337

338

339

340

341

342

343

344

345

346

347

348

349

350

351

352

353

354

355

356

357

358

359

containing MWCNTs + glucose, MWCNTs + lignin, MWCNTs + lignin + glucose, or MWCNTs alone (Figure 7; Figure S2 in supplementary material). Interestingly, in the samples from both fungi cultures grown on MWCNTs + glucose, MWCNTs + lignin, MWCNTs + lignin + glucose, worms survival significantly increased, comparing to that containing MWCNTs alone, being a clearer effect with the $P$. ostreatus fungus.

To deepen in the MWCNTs mechanism of toxicity involved, we determined the generation of reactive oxygen species (ROS) in the WT nematode strain. The results showed that the $C$. elegans WT increased the fluorescence dependent of anion superoxide amounts under the MWCNTs treatment (Figure 8A). This effect was further confirmed when the worm clone (CL2166) GFP oxidative stress-inducible was used, the fluorescence by GFP activity was significantly increased and observed in all the body of nematodes treated with MWCNTs (Figure $8 \mathrm{~A})$.

In addition, we observed that the CL2166 worm clone showed a basal fluorescence when it was exposed to fungal culture medium, also like adding lignin (Figure 8B). However, when

MWCTNs, MWCNTs + glucose, or MWCNTs + glucose + lignin were incinerated and added to the worm suspensions, their fluorescence significantly was increased (Figure 8C). Importantly, the intensity of fluorescence (ROS generation) in the worms was increased when used incinerated fungi culture medium from P. ostreatus and P. chrysogenum (grown for 28 days on a medium with MWCNTs), indicating increases of toxicity (ROS increase) on worms (Figure 8C). Quantitative determination of GFP-fluorescence in the worms corroborated the increase of ROS induction in the worms treated with the incinerated culture medium from both fungi (Figure 9).

\section{Discussion}

Nanotechnology is an emerging area of multidisciplinary interest because the extraordinary physical and chemical properties of nanoparticles (Zaytseva, 2016). The MWCNTs demand is in growing; however, its accumulation in the environment due to their high structural stability, represents great environment risks to consider, since MWCNTs have been associated with toxic effects in several biological systems (Ge et al. 2016; Shin et al. 2015). An alternative to biodegrade MWCNTs or reduce collateral environmental risks of these nanoparticles is the use of mycorremediation with ligninolytic fungi such as $P$. ostreatus and $P$. chrysogenum, which by synthesizing extracellular enzymes such as laccase, lignin-peroxidase (LiP), and manganeseperoxidase (MnP) represent an alternative to mitigate the ecological damage (Fuji et al. 2013; Janusz et al. 2013).

The use of white rot fungi to restore environments contaminated with highly recalcitrant organic xenobiotics have been widely documented, due to the ability of basidiomyces genus to synthesize high amounts of peroxidases and laccases (Fuji et al. 2013). P. ostreatus belongs to the Basidiomycetes group, which are ligninolytic fungi considered among the most efficient microorganisms to mineralize lignin. However, little has been documented on the enzymatic 
360

361

362

363

364

365

366

367

368

369

370

371

372

373

374

375

376

377

378

379

380

381

382

383

384

385

386

387

388

389

390

391

392

393

394

395

396

397

398

399

activity in deuteromycetes such as $P$. chrysogenum. In this work, we were able to demonstrate that lignin stimulates the activity of $\mathrm{LiP}$ and $\mathrm{MnP}$ in the $P$. chrysogenum cultures. In addition, it was observed that the presence of MWCNTs also influenced the stimulation of peroxidases activity in both the P. chrysogenum and P. ostreatus cultures (Figure 2A-C). It should be noted that the presence of MWCNTs or when supplemented with lignin triggered the activity of ligninolytic enzymes was triggered. The aforementioned result was in agreement with that reported in $T$. versicolor and $P$. tremellosa isolates wherein the presence of SWCNTs positively modified its lignocellulolytic activity (Berry et al. 2014). Relevantly, our findings indicate that both fungi species were capable to grow efficiently on medium supplemented with MWCNTs, demonstrating that this nanomaterial was no toxic at least to the concentration used $(10 \mu \mathrm{g} / \mathrm{mL}$ of MWCNTs) and an efficient fungal growth and ligninolytic enzymes excretion was observed. The results confirm that lignin played a key role in the induction of ligninolytic enzymes such as those reported in basidiomycetes during the lignin depolymerization process (Baltierra-Trejo et al. 2016; Cañas and Camarero 2010; Nousiainen et al. 2014).

Moreover, the changes in Raman bands indicated the occurrence of structural modifications in the surface of MWCNTs (Figure 3). The difference in the band IG band confirm de increment of defects in the crystalline structure of MWCNTs in the treatments with MWCNTs lignin and MWCNTs + lignin + glucose with both fungi, where the increase in height and width in this band, corresponds to the structural disorder in the graphitized materials (Liu et al. 2014). The changes in spectrum corresponding to the sp3 bonds of the distortions of the network in the curved graphene sheets or the ends of the carbon nanotubes (Lehman et al. 2011), and loss of crystallinity by oxidation of these nanoparticles, it can be attributed to ligninolytic enzymes, wherein activities clearly increased in the cultures during the assimilation of lignin. Thus, the changes in the IR spectra suggest that both the $P$. ostreatus and $P$. chrysogenum fungi modified the structural composition of MWCNTs. The presence of new functional groups, such as $\mathrm{COOH}$ or CO as described (Lehman et al. 2011), confirms that the excretion/production of ligninolytic enzymes provoked chemical modifications in the composition of the MWCNTs. Furthermore, the incorporated functional groups in the MWCNTs suggested that a biotransformation process occurred. Our results indicate that both fungi have the ability to degrade/mineralize the MWCNTs in a dependent manner on the available carbon source. Thus, decreases in the amount of MWCNTs observed in the HR-TEM sample observations and TGA were correlated with the Raman and IR spectroscopy analyses; thereby indicating that the MWCNTs sustained chemical and structural modifications during the fungi growth on culture media supplemented mainly with lignin. This indicated the occurrence of biotransformation and biodegradation/mineralization processes associated with the ligninolytic fungal enzymatic activities. As revealed by Liu et al. 2014 , the intensity of the 2D band is correlated with the number of graphene layers that constitute the structure of MWCNTs, thereby suggesting a reduction in the thickness of the MWCNTs following the growth of $P$. chrysogenum and $P$. ostreatus fungi on the cultures containing MWCNTs + lignin, suggesting that the role of lignin is the induction of peroxidases, which chemically modify the chemical composition of the MWCNTs material.

Peer) reviewing PDF | (2020:11:55553:2:0:NEW 19 Feb 2021) 
400

401

402

403

404

405

406

407

408

409

410

411

412

413

414

415

416

417

418

419

420

421

422

423

424

425

426

427

428

429

430

431

432

433

434

435

436

437

438

439

In addition, the results obtained with both fungi indicate that the structural transformations of MWCNTs induced by the fungal enzymes also in presence of lignin or glucose increase the toxic effects than when nonculture the fungi. It should be noted that diverse effects on the nematodes as our in vivo model are caused by the dried samples from the fungi cultures that contain MWCNTs. First, the MWCNTs can be modified by the fungus strains when used with additional carbon source, without apparent toxic effects over their growth. However, MWCNTs exhibits a certain level of toxicity for the nematodes ( $25 \%$ death at $72 \mathrm{~h}$ exposure). Second, the presence of other carbon sources such as lignin or glucose which induce ligninolytic enzymes increases its toxicity over the nematode model ( $\sim 50-70 \%$ death). This effect is potentially explained due to the chemical and structural modifications in the MWCNTs observed, such as have been described to occur in microbial communities (Ge et al. 2016; Wu et al. 2019). However, we can no to explain why the medium supplemented with MWCNTs + glucose was more toxic to the worms than MWCNTs alone, but after fungi growth is effect was reverted as expected (Figure 7).

These chemical and structural modifications are clearly demonstrated in the Raman, IR, and HRTEM analyses, which were in correlation with the results of the biological effects such as growth and peroxidases production. In agree with TGA results, $P$. ostreatus degraded MWCNTs more efficient than $P$. chrysogenum fungi, indicating that almost no residues of MWCNTs present on cultures from $P$. ostreatus induced lower mortality in $C$. elegans worms compared to the respective MWCNT amounts from $P$. chrysogenum samples (Figure 7). The toxic effect observed by the MWCNTs addition against the C. elegans nematode further confirms an exacerbated increment of ROS generation as a toxicity mechanism (Figures 8 and 9). This effect could be due to the structural modification by the $\mathrm{COOH}, \mathrm{CO}$, or $\mathrm{OH}$ radicals identified on the MWCNTs, which functional groups which have been widely described to increase the toxicity in nanomaterials (Magrez et al. 2006; Zhou et al. 2017).

In the study, the ability of fungi species such as $P$. ostreatus and $P$. chrysogenum to grow on a culture medium with the presence of highly recalcitrant nanoparticles (MWCNTs) was evidenced, which toxic effects of the MWCNTs over the fungi were not observed. Our findings suggest that the degree of toxicity of the synthetic MWCNTs in the in vivo nematode model was modified by the fungal laccase and peroxidases activity excreted to the culture media, and these correlated with chemical modifications observed. The toxic effect of the MWCNTs against the C. elegans nematode involved an increased ROS generation. Interestingly, the results obtained in the study indicate the potential ability of the fungi species $P$. ostreatus and $P$. chrysogenum to produce structural biotransformation on MWCNTs in a relatively short time, indicating that although the toxicity was increased under certain growth conditions, the amounts of the carbon nanomaterial was significantly decreased, as confirmed by spectroscopy analyses. The fact is mainly attributed to the ability of the fungi to synthesize ligninolytic enzymes, wherein extracellular enzymes are related with the assimilation mechanism of residual lignin and other

Peer] reviewing PDF | (2020:11:55553:2:0:NEW 19 Feb 2021) 
440 persistent or recalcitrant compounds. The findings indicated that the ligninolytic enzymes

441 induced by lignin are key in the chemical and structural modifications observed in the

442 MWCNTs, being these modifications important in the toxicity degree and environmental

443 persistence of these carbon nanomaterials.

444

445 Conclusions

446 The results obtained in the work further confirmed that the MWCNTs can be subject to

447 biotransformation events in the environment via the mycorremediation, thereby modify its toxic

448 effects over others organisms. This study contributes with a better understanding of the

449 interactions between engineered carbon nanomaterials and microbial communities, as their

450 beneficial as toxicological effects over the ecosystem.

451

452

453

454

455

456

457

458

459

460

461

462

463

464

465

466

467

468

469

470

471

472

473

474

475

476

477

478

479

480

481

482

\section{Acknowledgements}

C. elegans strains were provided to J. Altamirano and A. Guzmán by the Caenorhabditis Genetics Center (University of Minnesota, Minneapolis, MN, USA).

\section{References}

Al-Rekaby LS. 2018. Influence of Multiwalled Carbon Nanotubes and Biostimulators on Growth and Content of Bioactive Constituents of Karkade (Hibiscus sabdariffa L.). Journal of Botany. 9097363. DOI 10.1155/2018/9097363.

Allen BL, Kotchey GP, Chen Y, Yanamala NVK, Klein-seetharaman J, Kagan VE, and Star A. 2009. Mechanistic Investigations of Horseradish Peroxidase-Catalyzed Degradation of Single-Walled Carbon Nanotubes. American Chemical Society:17194-17205. DOI 10.1021/ja9083623.

Andõn FT, Kapralov AA, Yanamala N, Feng W, Baygan A, Chambers BJ, Hultenby K, Ye F, Toprak MS, Brandner BD, Fornara A, Klein-Seetharaman J, Kotchey GP, Star A, Shvedova AA, Fadeel B, and Kagan VE. 2013. Biodegradation of single-walled carbon nanotubes by eosinophil peroxidase. Small 9:2721-2729. DOI 10.1002/smll.201202508.

Baltierra-Trejo E, Silva-Espino E, Márquez-Benavides L, and Sánchez-Yáñez JM. 2016. Inducción de la degradación de lignina de paja de trigo en aromáticos por Aspergillus spp. y Penicillium chrysogenum. Journal of the Selva Andina Research Society 7:10-19. ISSN 2072-9308 Online https://www.researchgate.net/publication/308024493

Barr DP, Aust SD. 1994. Pollutant degradation by white rot fungi. Rev Environ Contam Toxicol.138:49-72. DOI: 10.1007/978-1-4612-2672-7_3.

Berry TD, Filley TR, and Blanchette Ra. 2014. Oxidative enzymatic response of white-rot fungi to single-walled carbon nanotubes. Environmental Pollution 193:197-204. DOI 10.1016/j.envpol.2014.06.013.

Bhattacharya K, Andón FT, El-sayed R, and Fadeel B. 2013. Mechanisms of carbon nanotubeinduced toxicity: Focus on pulmonary in $\mathrm{fl}$ ammation. Advanced Drug Delivery Reviews 65:2087-2097. DOI: 10.1016/j.addr.2013.05.012. 
483

484

485

486

487

488

489

490

491

492

493

494

495

496

497

498

499

500

501

502

503

504

505

506

507

508

509

510

511

512

513

514

515

516

517

518

519

520

521

522

523

524

525

526

527

Burlaka OM, Pirko YV, Yemets AI, and Blume YB. 2015. Plant genetic transformation using carbon nanotubes for DNA delivery. Cytology and Genetics 49:349-357. DOI 10.3103/S009545271506002X.

Cañas AI, and Camarero S. 2010. Laccases and their natural mediators: Biotechnological tools for sustainable eco-friendly processes. Biotechnology Advances 28:694-705. DOI 10.1016/j.biotechadv.2010.05.002.

Chandrasekaran G, Choi SK, Lee YC, Kim GJ, and Shin HJ. 2014. Oxidative biodegradation of single-walled carbon nanotubes by partially purified lignin peroxidase from Sparassis latifolia mushroom. Journal of Industrial and Engineering Chemistry 20:3367-3374. DOI 10.1016/j.jiec.2013.12.022.

Chen M, Zeng G, Tan Z, Jiang M, Li H, Liu L, Zhu Y, Yu Z, Wei Z, Liu Y, and Xie G. 2011. Understanding lignin-degrading reactions of ligninolytic enzymes: Binding affinity and interactional profile. PLOS ONE 6. DOI 10.1371/journal.pone.0025647.

Chen P-H, Hsiao K-M, and Chou C-C. 2013. Molecular characterization of toxicity mechanism of single-walled carbon nanotubes. Biomaterials 34:5661-5669. DOI 10.1016/j.biomaterials.2013.03.093.

De Volder MFL, Tawfick SH, Baughman RH, and Hart aJ. 2013. Carbon nanotubes: present and future commercial applications. Science (New York, NY) 339:535-539. DOI 10.1126/science. 1222453.

Fujii K, Uemura M, Hayakawa C, Funakawa S, and Kosaki T. 2013. Environmental control of lignin peroxidase, manganese peroxidase, and laccase activities in forest floor layers in humid Asia. Soil Biology and Biochemistry 57:109-115. DOI 10.1016/j.soilbio.2012.07.007.

Gan S, Lau EV, and Ng HK. 2009. Remediation of soils contaminated with polycyclic aromatic hydrocarbons (PAHs). Journal of Hazardous Materials 172:532-549. DOI 10.1016/j.jhazmat.2009.07.118.

Gao HJ, Wang YW, Zhang WT, Wang WL, and Mu ZM. 2011. Isolation, identification and application in lignin degradation of an ascomycete GHJ-4. African Journal of Biotechnology 10(20):4166-4174. DOI 10.5897/AJB10.2250.

Ge Y, Priester JH, Mortimer M, Chang CH, Ji Z, Schimel JP, and Holden PA. 2016. Long-Term Effects of Multiwalled Carbon Nanotubes and Graphene on Microbial Communities in Dry Soil. Environmental Science \& Technology 50:3965-3974. DOI 10.1021/acs.est.5b05620.

Haritash AK, and Kaushik CP. 2009. Biodegradation aspects of Polycyclic Aromatic Hydrocarbons (PAHs): A review. Journal of Hazardous Materials 169:1-15. DOI 10.1016/j.jhazmat.2009.03.137.

Ibrahim V, Mendoza L, Mamo G, and Hatti-Kaul R. 2011. Blue laccase from Galerina sp.: Properties and potential for Kraft lignin demethylation. Process Biochemistry 46:379384. DOI 10.1016/j.procbio.2010.07.013.

Janusz G, Kucharzyk KH, Pawlik A, Staszczak M, and Paszczynski AJ. 2013. Fungal laccase, manganese peroxidase and lignin peroxidase: Gene expression and regulation. Enzyme and Microbial Technology 52:1-12. DOI 10.1016/j.enzmictec.2012.10.003.

Kagan VE, Konduru NV, Feng W, Allen BL, Conroy J, Volkov Y, Vlasova II, Belikova NA, Yanamala N, Kapralov A, Tyurina YY, Shi J, Kisin ER, Murray AR, Franks J, Stolz D, Gou P, Klein-Seetharaman J, Fadeel B, Star A, and Shvedova AA. 2010. Carbon 
528

529

530

531

532

533

534

535

536

537

538

539

540

541

542

543

544

545

546

547

548

549

550

551

552

553

554

555

556

557

558

559

560

561

562

563

564

565

566

567

568

569

570

571

572

nanotubes degraded by neutrophil myeloperoxidase induce less pulmonary inflammation. Nature Nanotechnology 5:354-359. DOI 10.1038/nnano.2010.44.

Lara-Romero J, Campos-García J, Dasgupta-Schubert N, Borjas-García S, Tiwari DK, ParaguayDelgado F, Jiménez-Sandoval S, Alonso-Nuñez G, Gómez-Romero M, Lindig-Cisneros R, Reyes De la Cruz H, and Villegas JA. 2017. Biological effects of carbon nanotubes generated in forest wildfire ecosystems rich in resinous trees on native plants. PeerJ 5:e3658-e3658. DOI 10.7717/peerj.3658.

Lehman JH, Terrones M, Mansfield E, Hurst KE, and Meunier V. 2011. Evaluating the characteristics of multiwall carbon nanotubes. Carbon 49:2581-2602. DOI 10.1016/j.carbon.2011.03.028.

Leung CK, Wang Y, Malany S, Deonarine A, Nguyen K, Vasile S, Choe KP. 2013. An ultra high-throughput, whole-animal screen for small molecule modulators of a specific genetic pathway in Caenorhabditis elegans. PLoS One. 8(4): e62166. DOI 10.1371/journal.pone.0062166.

Lin C, Fugetsu B, Su Y, and Watari F. 2009. Studies on toxicity of multi-walled carbon nanotubes on Arabidopsis T87 suspension cells. Journal of Hazardous Materials 170:578-583. DOI 10.1016/j.jhazmat.2009.05.025.

Liu W-W, Chai S-P, Mohamed AR, and Hashim U. 2014. Synthesis and characterization of graphene and carbon nanotubes: A review on the past and recent developments. Journal of Industrial and Engineering Chemistry 20:1171-1185. DOI 10.1016/j.jiec.2013.08.028.

Ma Q, Yilihamu A, Ming Z, Yang S, Shi M, Ouyang B, Zhang Q, Guan X, Yang ST. 2019. Biotransformation of Pristine and Oxidized Carbon Nanotubes by the White Rot Fungus Phanerochaete chrysosporium. Nanomaterials (Basel). 19;9(9):1340. DOI: 10.3390/nano9091340. PMID: 31546834; PMCID: PMC6781511.

Magrez A, Kasas S, Salicio V, Pasquier N, Seo JW, Celio M, Catsicas S, Schwaller B, and Forro L. 2006. Cellular toxicity of carbon-based nanomaterials. Nano Letters 6:1121-1125. DOI 10.1021/n1060162e.

Modugno G, Ksar F, Battigelli A, Russier J, Lonchambon P, Eleto Da Silva E, Ménard-Moyon C, Soula B, Galibert AM, Pinault M, Flahaut E, Mayne-L'Hermite M, and Bianco A. 2016. A comparative study on the enzymatic biodegradability of covalently functionalized double- and multi-walled carbon nanotubes. Carbon 100:367-374. DOI 10.1016/j.carbon.2016.01.023.

Nousiainen P, Kontro J, Manner H, Hatakka A, and Sipilä J. 2014. Phenolic mediators enhance the manganese peroxidase catalyzed oxidation of recalcitrant lignin model compounds and synthetic lignin. Fungal Genetics and Biology 72:137-149. DOI 10.1016/j.fgb.2014.07.008.

Palmieri G, Giardina P, Scaloni A, Capasso A, Sannia G, and Bianco C. 1997. PROTEIN CHEMISTRY AND STRUCTURE : A Novel White Laccase from Pleurotus ostreatus A Novel White Laccase from Pleurotus ostreatus. The Journal of Biological Chemistry 272:31301-31307. DOI 10.1074/jbc.272.50.3130.

Parks AN, Chandler GT, Ho KT, Burgess RM, and Ferguson PL. 2015. Environmental biodegradability of [14 C] single-walled carbon nanotubes by Trametes versicolor and natural microbial cultures found in New Bedford Harbor sediment and aerated wastewater treatment plant sludge. Environmental Toxicology and Chemistry 34:247-251. DOI 10.1002/etc. 2791. 
573 Petersen EJ, Huang Q, and Weber WJ. 2008. Ecological uptake and depuration of carbon

574

575

576

577

578

579

580

581

582

583

584

585

586

587

588

589

590

591

592

593

594

595

596

597

598

599

600

601

602

603

604

605

606

607

608

609

610

611

612

613

614

615

616

617

618 nanotubes by Lumbriculus variegatus. Environmental Health Perspectives 116:496-500. DOI 10.1289/ehp.10883.

Petersen EJ, Zhang L, Mattison NT, O'Carroll DM, Whelton AJ, Uddin N, Nguyen T, Huang Q, Henry TB, Holbrook RD, and Chen KL. 2011. Potential release pathways, environmental fate, and ecological risks of carbon nanotubes. Environmental Science and Technology 45:9837-9856. DOI 10.1021/es201579y.

Qu X, Alvarez PJJ, Li Q. 2013. Applications of nanotechnology in water and wastewater treatment. Water Res. 47(12):3931-46. DOI 10.1016/j.watres.2012.09.058.

Rong Hong, Wang Chengrun, Yu Xiaorui, Fan Jiang, Wang Yuchauan, Gan Xianqing, Wan Yun. 2018. Carboxylated multi-walled carbon nanotubes exacerbated oxidative damage in roots of Vicia faba L. seedlings under combined stress of lead and cadmium. Ecotoxicology and Environmental Safety. 161:616-623. DOI 10.1016/j.ecoenv.2018.06.034.

Saifuddin N, Raziah AZ, and Junizah AR. 2013. Carbon nanotubes: A review on structure and their interaction with proteins. Journal of Chemistry. DOI 10.1155/2013/676815.

Shin S, Song I, and Um S. 2015. Role of Physicochemical Properties in Nanoparticle Toxicity. Nanomaterials 5:1351-1365. DOI 10.3390/nano5031351.

Stiernagle T. 2006. Maintenance of C. elegans: WormBook, ed. The C. elegans Research Community, WormBook, DOI 10.1895/wormbook.1.101.1. http://www.wormbook.org.

Tan XM, Lin C, and Fugetsu B. 2009. Studies on toxicity of multi-walled carbon nanotubes on suspension rice cells. Carbon 47:3479-3487. DOI 10.1016/j.carbon.2009.08.018.

Ting WTE, Yuan SY, Wu SD, and Chang BV. 2011. Biodegradation of phenanthrene and pyrene by Ganoderma lucidum. International Biodeterioration and Biodegradation 65:238-242. DOI 10.1016/j.ibiod.2010.11.007

Uo M, Akasaka T, Watari F, Sato Y, and Tohji K. 2011. Toxicity evaluations of various carbon nanomaterials. Dental Materials Journal 30:245-263. DOI 10.4012/dmj.2010-039.

Vlasova II, Vakhrusheva TV, Sokolov AV, Kostevich VA, and Ragimov AA. 2011. Peroxidaseinduced degradation of single-walled carbon nanotubes: hypochlorite is a major oxidant capable of in vivo degradation of carbon nanotubes. Journal of Physics: Conference Series 291:012056-012056. DOI 10.1088/1742-6596/291/1/012056.

Wang C, Sun H, Li J, Li Y, Zhang Q. 2009. Enzyme activities during degradation of polycyclic aromatic hydrocarbons by white rot fungus Phanerochaete chrysosporium in soils. Chemosphere 77(6):733-8. DOI 10.1016/j.chemosphere.2009.08.028.

Wu F, You Y, Zhang X, Zhang H, Chen W, Yang Y, Werner D, Tao S, and Wang X. 2019. Effects of Various Carbon Nanotubes on Soil Bacterial Community Composition and Structure. Environmental Science \& Technology. DOI 10.1021/acs.est.8b06909.

Yadav T, Mungray AA, and Mungray AK. 2016. Effect of multiwalled carbon nanotubes on UASB microbial consortium. Environmental Science and Pollution Research 23:40634072. DOI 10.1007/s11356-015-4385-y.

Zaytseva O. 2016. Differential Impact of Multi-Walled Carbon Nanotubes on Germination and Seedling Development of Glycine Max, Phaseolus vulgaris and Zea mays. European chemmiscal Bulletin 5:202-210 DOI 10.17628/ECB.2016.5.202.

Zhang C, Chen W, and Alvarez PJJ. 2014. Manganese peroxidase degrades pristine but not surface-oxidized (carboxylated) single-walled carbon nanotubes. Environmental Science and Technology 48:7918-7923. DOI 10.1021/es5011175. 
619 Zhao Y, Allen BL, and Star A. 2012. Enzymatic Degradation of Multi-Walled Carbon $620 \quad$ Nanotubes. Journal of Physics and chemistry 115:9536-9544. DOI

$621 \quad 10.1021 / j p 112324 d$.Enzymatic.

622 Zhou L, Forman HJ, Ge Y, and Lunec J. 2017. Multi-walled carbon nanotubes: A cytotoxicity 623 study in relation to functionalization, dose and dispersion. Toxicol In Vitro 42:292-298. $624 \quad$ DOI 10.1016/j.tiv.2017.04.027.

625 


\section{Figure legends}

627

628

629

630

631

632

633

634

635

636

637

638

639

640

641

642

643

644

645

646

647

648

649

650

651

652

653

654

655
Figure 1. Growth of $P$. chrysogenum and P. ostreatus on medium supplemented with MWCNTs. Growth assays were carried out in liquid medium supplemented with MWCNTs $(10 \mu \mathrm{g} / \mathrm{mL})$ at $30^{\circ} \mathrm{C}$ incubation by 28 days. Fungi growth is showed as biomass, determined by dry weight of culture media (g/L). (A) P. chrysogenum, (B) P. ostreatus. Results are the means of triplicate assays. SE values are indicated as bars.

Figure 2. Ligninolytic activities on cultures of $P$. chrysogenum and $P$. ostreatus grown in presence of MWCNTs. Fungi growth was carried out in liquid medium supplemented with MWCNTs $(10 \mu \mathrm{g} / \mathrm{mL})$ at $30^{\circ} \mathrm{C}$ incubation by 28 days. Effect of carbon sources on ligninolytic activities at 28-day of culture of $P$. chrysogenum (A-C) and P. ostreatus (D-F) fungi. Enzyme activity units are determined by dry weight from $100 \mathrm{~mL}$ of culture media. Results are the means of three independent assays. SE values are indicated as bars.

Figure 3. Chemical modifications into the MWCNTs by $P$. ostreatus and $P$. chrysogenum growth determined by Raman spectroscopy. Samples of fugal cultures supplemented with MWCNTs were dried and incinerated prior to Raman analysis. Representative Raman scattering spectra (He-Ne laser emitting at $514 \mathrm{~nm}$ ) of the culture samples are shown; MWCNTs at the beginning of the assay ( 0 day, black lines) and cultures samples after 28 days of incubation (red lines). Characteristic bands of MWCNTs are shown: ID band $\left(\sim 1370 \mathrm{~cm}^{-1}\right)$, IG band $\left(\sim 1600 \mathrm{~cm}^{-1}\right)$, and 2D $\left(\mathrm{G}^{\prime}\right)$ band $\left(\sim 2640 \mathrm{~cm}^{-1}\right)$.

Figure 4. Chemical modifications into the MWCNTs by $P$. ostreatus and $P$. chrysogenum growth determined by FTIR spectroscopy. Samples of fugal cultures supplemented with MWCNTs were dried and incinerated prior to FTIR analysis. Cultures with MWCNTs grown with the $P$. ostreatus (red line) and P. chrysogenum (blue line) analyzed after 28 days of incubation. Controls are showed (black and light blue lines). Signals of radicals or functional groups are indicated. Results were conducted by triplicate and representative graph is shown. 
656

657

658

659

660

661

662

663

664

665

666

667

668

669

670

671

672

673

674

675

676

677

678

679

680

681

682

683

684

685

686

687
Figure 5. Structural modifications into the MWCNTs by P. ostreatus and P. chrysogenum growth observed by HR-TEM. Samples of fugal cultures supplemented with MWCNTs were dried, incinerated and mounted on perforated carbon-coated $\mathrm{Cu}^{\mathrm{o}}$ grid to observed by transmission electronic microscopy. P. ostreatus fungus cultures incubated with (A) MWCNTs for 0 days, and after 28 days of incubation (B), (C) MWCNTs + glucose at 28 day, (D) MWCNTs + lignin at 28 day, and (E) MWCNTs lignin + glucose at 28 day. $P$. chrysogenum fungus cultures incubated with (F) MWCNTs for 0 days, and after 28 days of incubation with MWCNTs (G), (H) MWCNTs + glucose at 28 day, (I) MWCNTs + lignin at 28 day, and (J) MWCNTs + lignin + glucose at 28 day. Increased-amplification HRTEM images of $P$. ostreatus fungus cultures incubated with MWCNTs + lignin at 0 day $(\mathrm{K}-\mathrm{M})$ and cultured with MWCNTs + lignin at 28 day $(\mathrm{N}-\mathrm{O})$. MWCNTs aggregates and nanotube structures are indicated with arrows.

Figure 6. Mass loss on cultures of $P$. ostreatus and $P$. chrysogenum grown with MWCNTs by thermogravimetric analyses (TGA). Samples of fugal cultures supplemented with MWCNTs were dried prior to TGA analysis. Before incubation (0 days) and after 28 days. $P$. ostreatus and P. chrysogenum cultures added with MWCNTs were analyzed after 28 days of cultivation. Representative plots are shown.

Figure 7. Toxic effects on $C$. elegans worms exposed to cultures of $P$. ostreatus and $P$. chrysogenum grown with MWCNTs. Samples from media supplemented with MWCNTs and from fugal cultures grown by 28 days were dried and incinerated prior to worms survival test. Adult $C$. elegans worms (20 individuals) were incubated in $1 \mathrm{~mL}$ of culture media (control) or residues obtained after incineration at $300{ }^{\circ} \mathrm{C}$ of samples from the $P$. ostreatus and $P$. chrysogenum fungi grown on medium with MWCNTs. Worms survival was determined at $72 \mathrm{~h}$ of exposure as described in Materials and Methods. Conditions of treatment are indicated. Bars represent the mean value \pm standard error (SE) of three independent assays with $\mathrm{n}=20$ for each treatment. One-way ANOVA analysis was carried out, with Bonferroni post-hoc test. Values for $P<0.05$ are shown with different lower-case letters. Kinetics of worm survival are showed in Figure S2 of supplementary material. 
688

689

690

691

692

693

694

695

696

697

698

699

700

701

702

703

704

705

706

707

708

709

Figure 8. Microscopy fluorescence images of C. elegans worms treated with MWCNTs. A) C. elegans WT treated with $10 \mu \mathrm{g} / \mathrm{mL}$ MWCNTs and superoxide generation was developed by using the Dihydroethidium fluorescent probe (DHE). (i) Worms observed with clear field at 10X magnification. (ii) Worms treated with MWCNTs by 4 h exposition and using DHE as probe, after observed by fluorescent field at $10 \mathrm{X}$ magnification. $C$. elegans (CL2166) GFP oxidative stress-inducible clone treated with $10 \mu \mathrm{g} / \mathrm{mL}$ MWCNTs; (iii) clear field image and (iv) fluorescent field at 10X magnification. B) C. elegans CL2166-GFP treated without or with lignin as indicated. C) C. elegans CL2166-GFP worms treated with samples taken from culture media of the $P$. ostreatus and $P$. chrysogenum fungi grown on medium with $10 \mu \mathrm{g} / \mathrm{mL}$ MWCNTs as indicated. Samples of fugal cultures were dried and incinerated prior to test. Worms observed by fluorescent field at 10X magnification. Representative images are shown.

Figure 9. Reactive oxygen species production determined by fluorescence on the $C$. elegans CL2166-GFP worms treated with MWCNTs. Worms treated by $24 \mathrm{~h}$ with the compounds at concentrations used in the culture media are indicated as control. Worms exposed to incinerated samples from fungi cultures grown on medium with MWCNTs are indicated as from $P$. ostreatus and $P$. chrysogenum. Fluorescence was determined using the Image $\mathrm{J}$ software in at least 10 worms of each treatment as observed in imagens showed in figure $8 \mathrm{C}$,. Bars represent the mean value \pm standard error $(\mathrm{SE}), \mathrm{n}=10$ for each treatment. One-way ANOVA analysis was carried out, with Tukey's post-hoc test. Values for $P<$ 0.05 are shown with different lower-case letters. 


\section{Figure 1}

Growth of $P$. chrysogenum and $P$. ostreatus on medium supplemented with MWCNTs.

Growth assays were carried out in liquid medium supplemented with MWCNTs $(10 \mu \mathrm{g} / \mathrm{mL})$ at $30{ }^{\circ} \mathrm{C}$ incubation by 28 days. Fungi growth is showed as biomass, determined by dry weight of culture media (g/L). (A) P. chrysogenum, (B) P. ostreatus. Results are the means of triplicate assays. SE values are indicated as bars. 

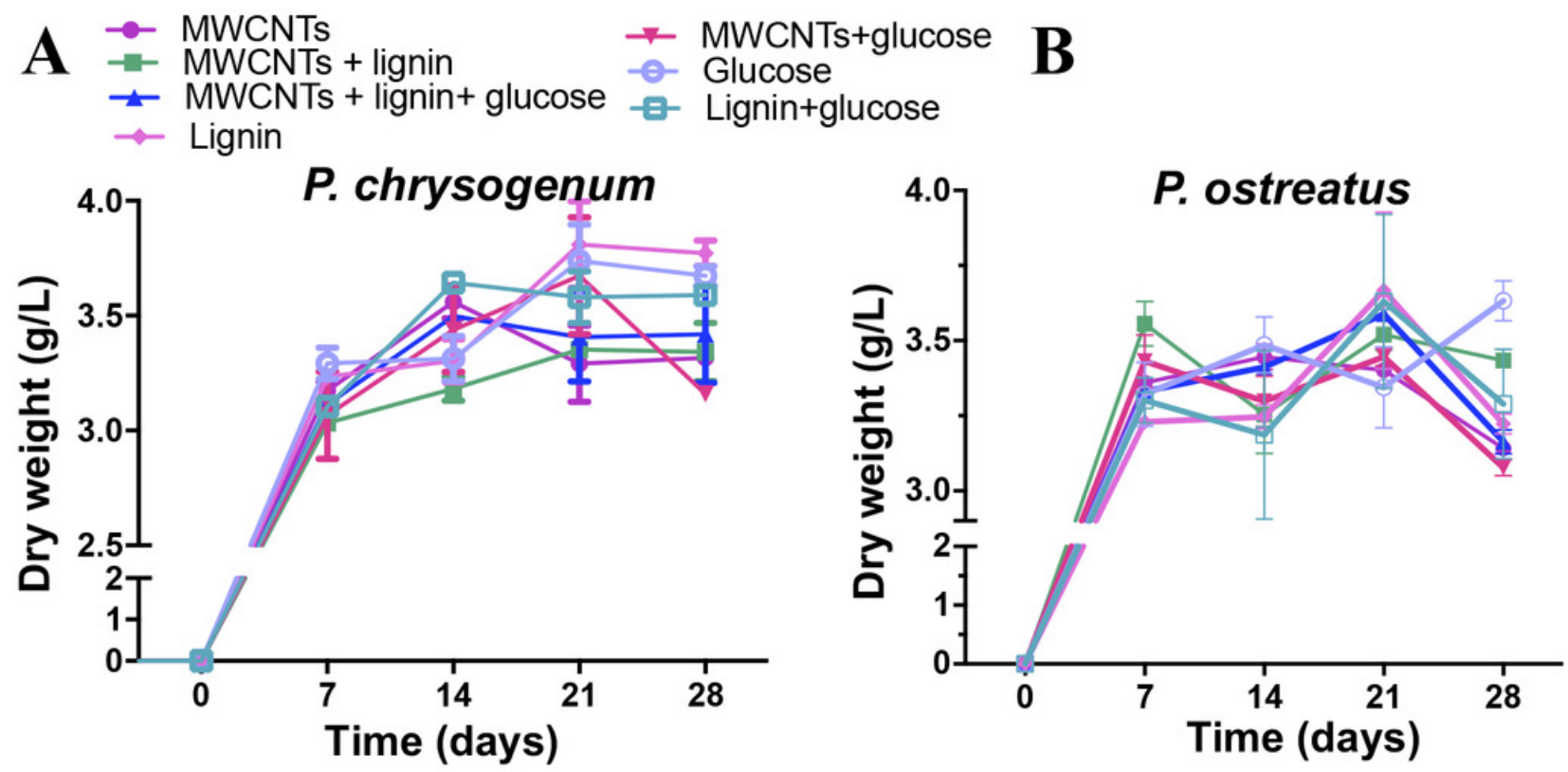


\section{Figure 2}

Ligninolytic activities on cultures of $P$. chrysogenum and $P$. ostreatus grown in presence of MWCNTs.

Fungi growth was carried out in liquid medium supplemented with MWCNTs $(10 \mu \mathrm{g} / \mathrm{mL})$ at 30

${ }^{\circ} \mathrm{C}$ incubation by 28 days. Effect of carbon sources on ligninolytic activities at 28-day of culture of $P$. chrysogenum (A-C) and $P$. ostreatus (D-F) fungi. Enzyme activity units are determined by dry weight from $100 \mathrm{~mL}$ of culture media. Results are the means of three independent assays. SE values are indicated as bars. 


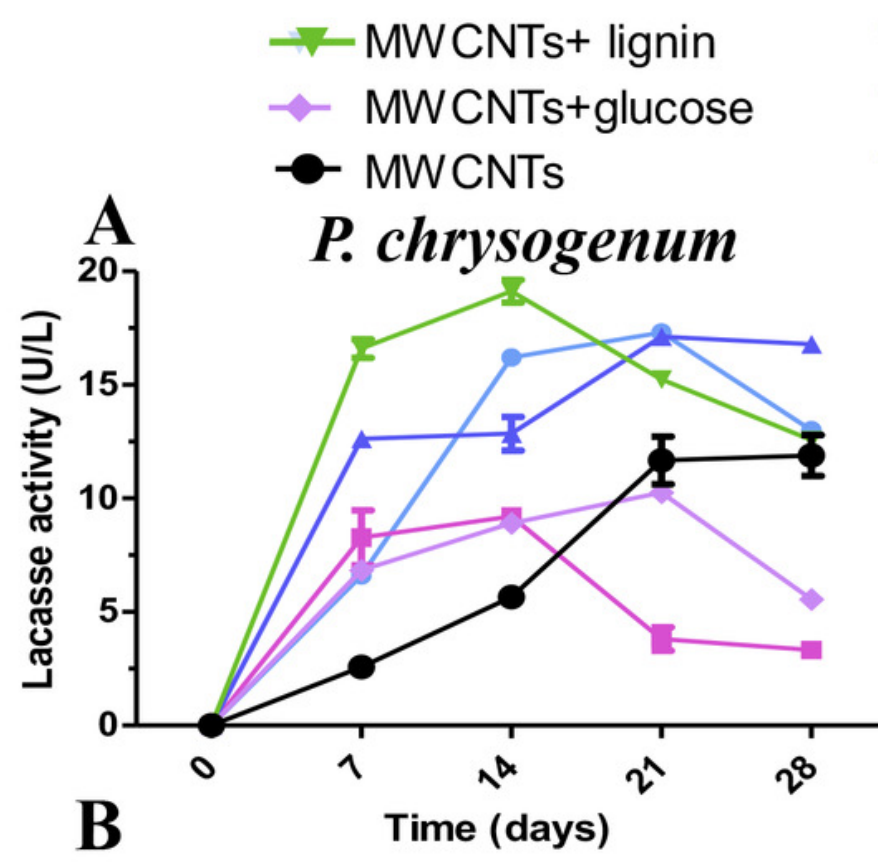

$\rightarrow$ Lignin

-느- Glucose

$\leftarrow$ MWCNTs+ lignin+glucose
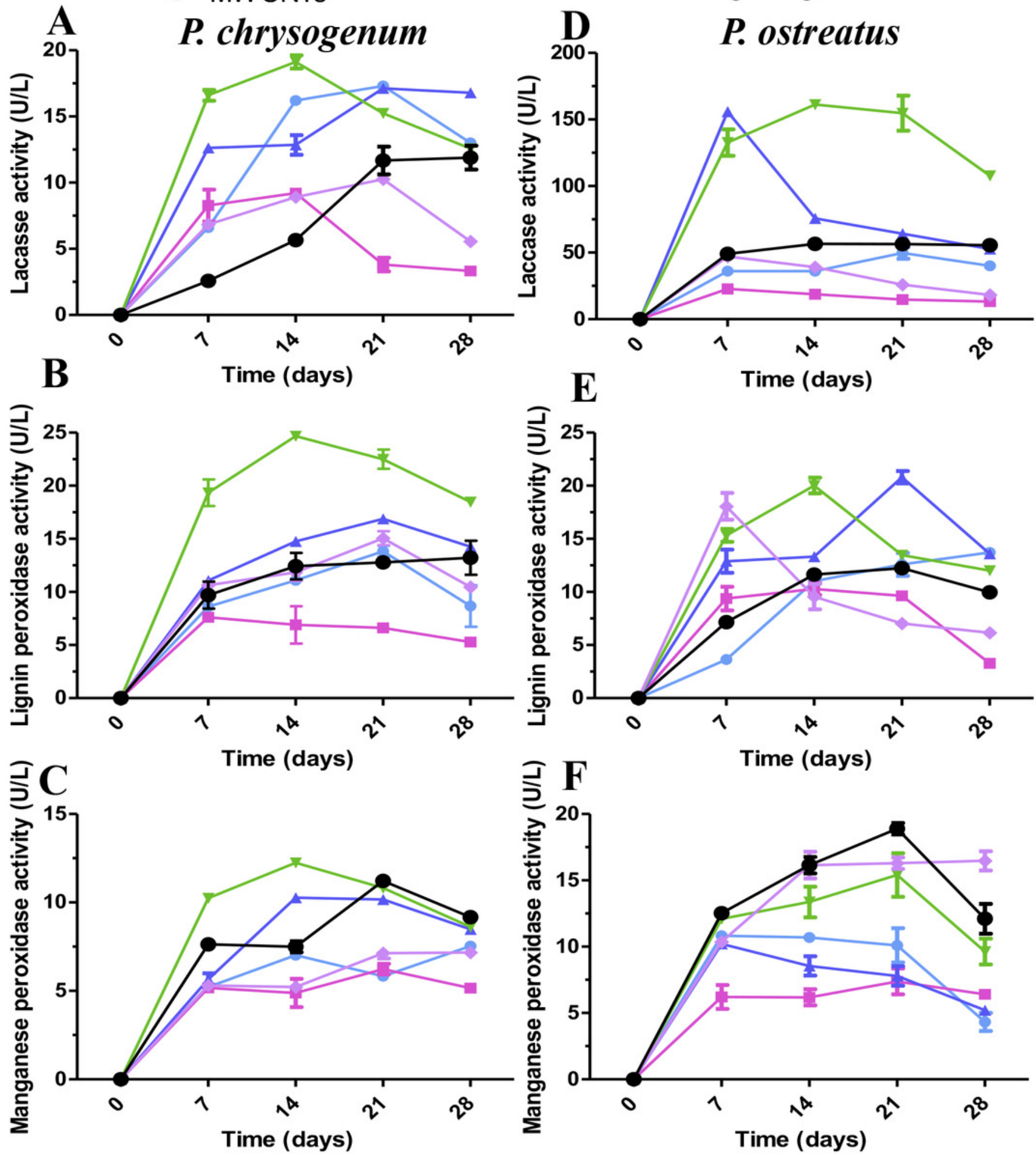


\section{Figure 3}

Chemical modifications into the MWCNTs by $P$. ostreatus and $P$. chrysogenum growth determined by Raman spectroscopy.

Samples of fugal cultures supplemented with MWCNTs were dried and incinerated prior to Raman analysis. Representative Raman scattering spectra (He-Ne laser emitting at $514 \mathrm{~nm}$ ) of the culture samples are shown; MWCNTs at the beginning of the assay (0 day, black lines) and cultures samples after 28 days of incubation (red lines). Characteristic bands of MWCNTs are shown: ID band $\left(\sim 1370 \mathrm{~cm}^{-1}\right)$, IG band $\left(\sim 1600 \mathrm{~cm}^{-1}\right)$, and 2D $\left(\mathrm{G}^{\prime}\right)$ band $\left(\sim 2640 \mathrm{~cm}^{-1}\right)$. 

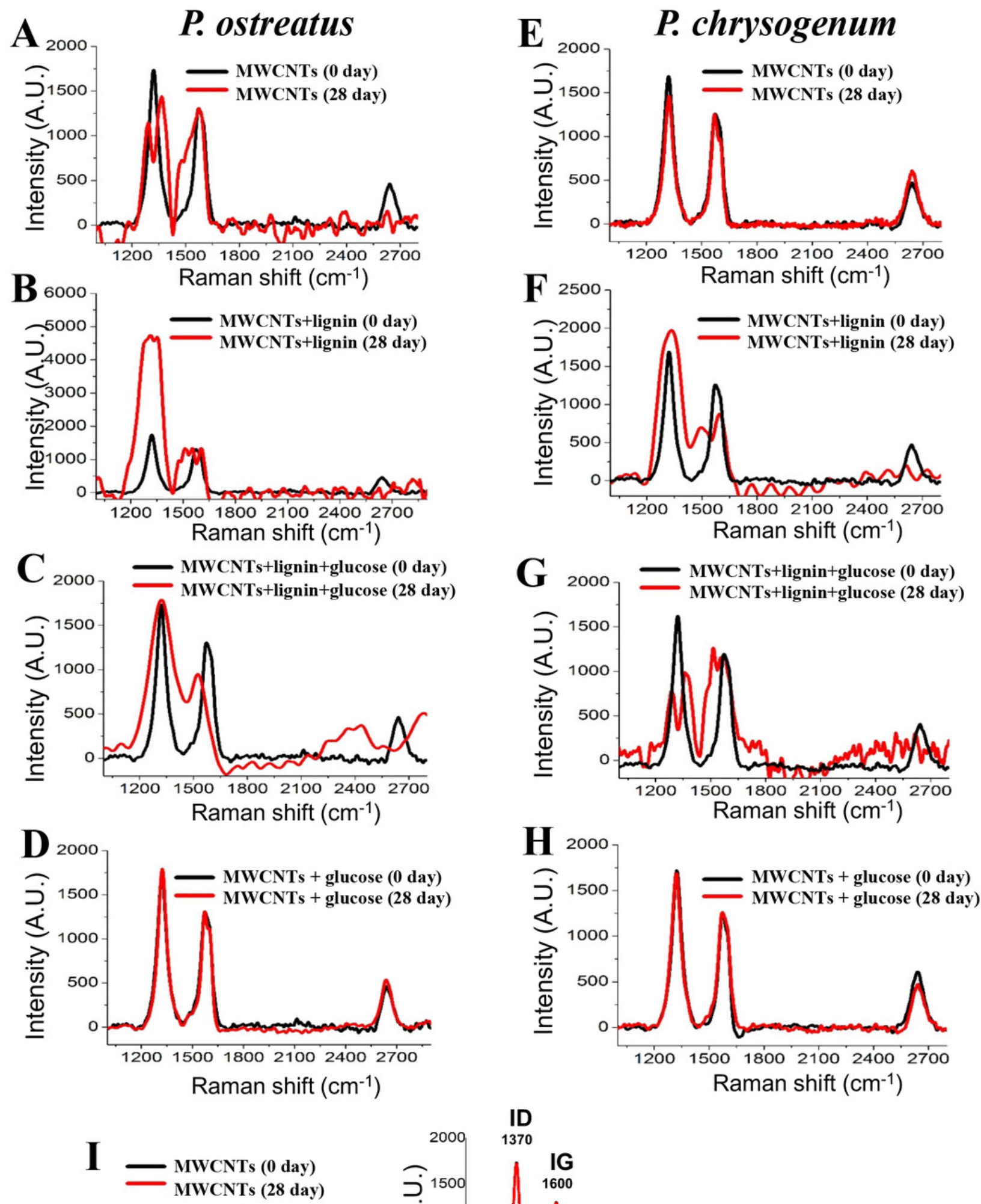

(without fungi exposition)

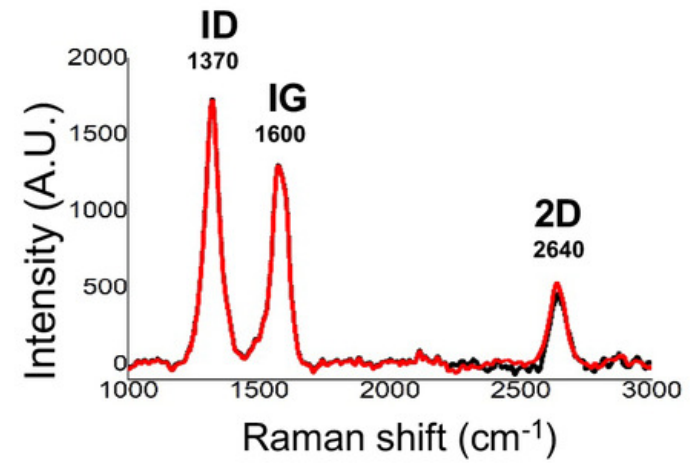




\section{Figure 4}

Chemical modifications into the MWCNTs by $P$. ostreatus and $P$. chrysogenum growth determined by FTIR spectroscopy.

Samples of fugal cultures supplemented with MWCNTs were dried and incinerated prior to FTIR analysis. Cultures with MWCNTs grown with the $P$. ostreatus (red line) and $P$. chrysogenum (blue line) analyzed after 28 days of incubation. Controls are showed (black and light blue lines). Signals of radicals or functional groups are indicated. Results were conducted by triplicate and representative graph is shown. 


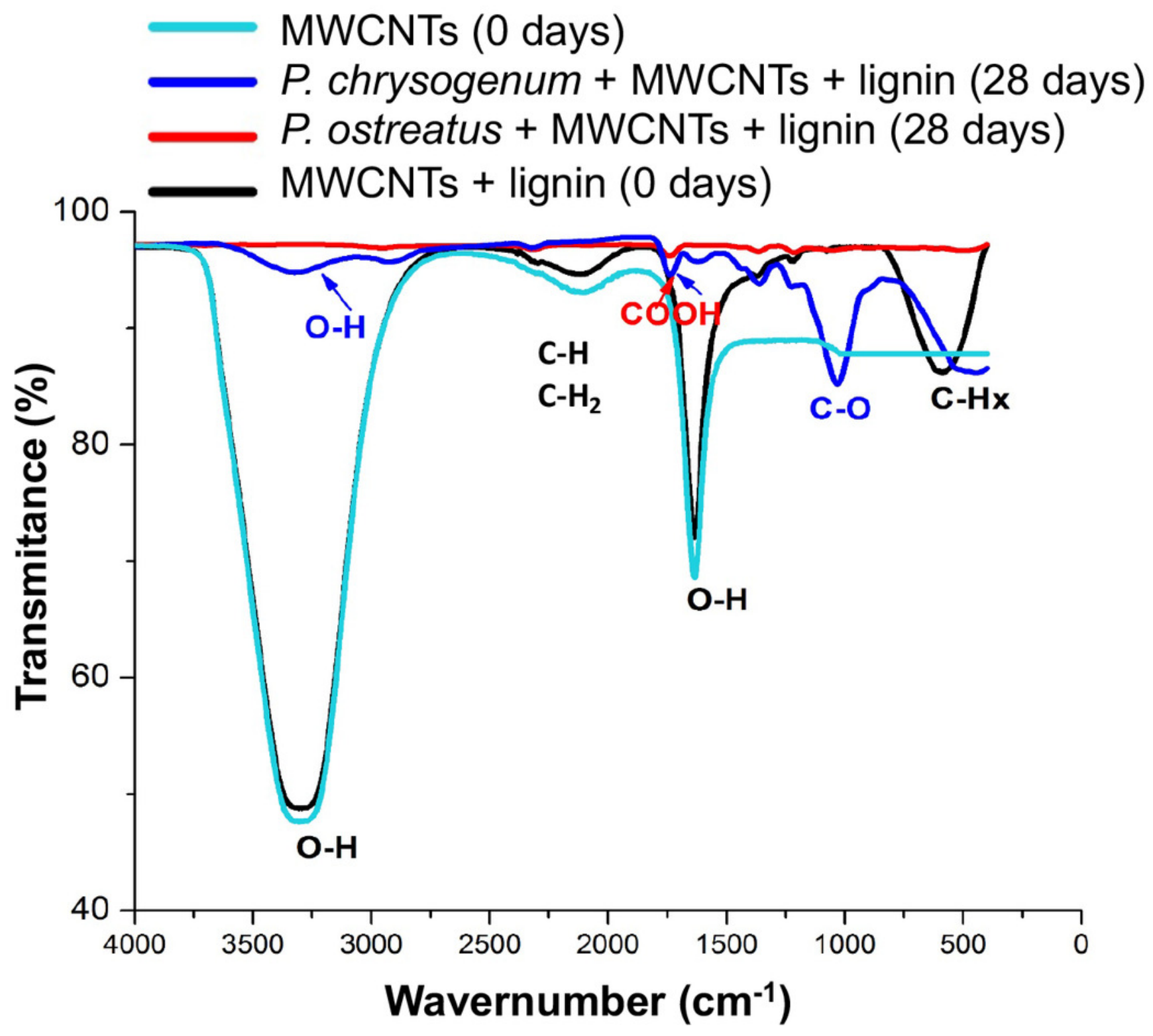




\section{Figure 5}

Structural modifications into the MWCNTs by $P$. ostreatus and $P$. chrysogenum growth observed by HR-TEM.

Samples of fugal cultures supplemented with MWCNTs were dried, incinerated and mounted on perforated carbon-coated $\mathrm{Cu}^{\circ}$ grid to observed by transmission electronic microscopy. $P$. ostreatus fungus cultures incubated with (A) MWCNTs for 0 days, and after 28 days of incubation (B), (C) MWCNTs + glucose at 28 day, (D) MWCNTs + lignin at 28 day, and (E) MWCNTs lignin + glucose at 28 day. P. chrysogenum fungus cultures incubated with (F) MWCNTs for 0 days, and after 28 days of incubation with MWCNTs (G), (H) MWCNTs + glucose at 28 day, (I) MWCNTs + lignin at 28 day, and (J) MWCNTs + lignin + glucose at 28 day. Increased-amplification HR-TEM images of $P$. ostreatus fungus cultures incubated with MWCNTs + lignin at 0 day (K-M) and cultured with MWCNTs + lignin at 28 day (N-O). MWCNTs aggregates and nanotube structures are indicated with arrows. 


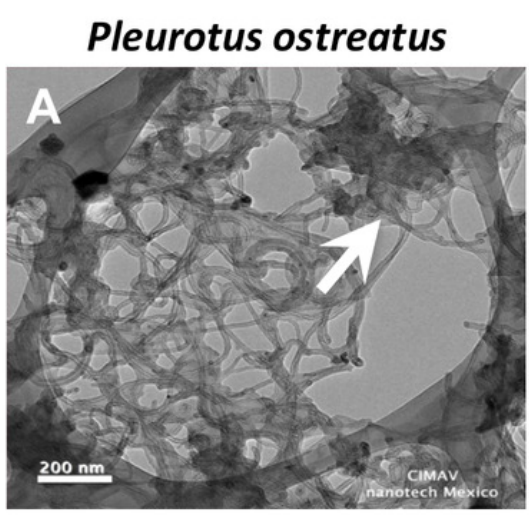

Penicillium chrysogenum
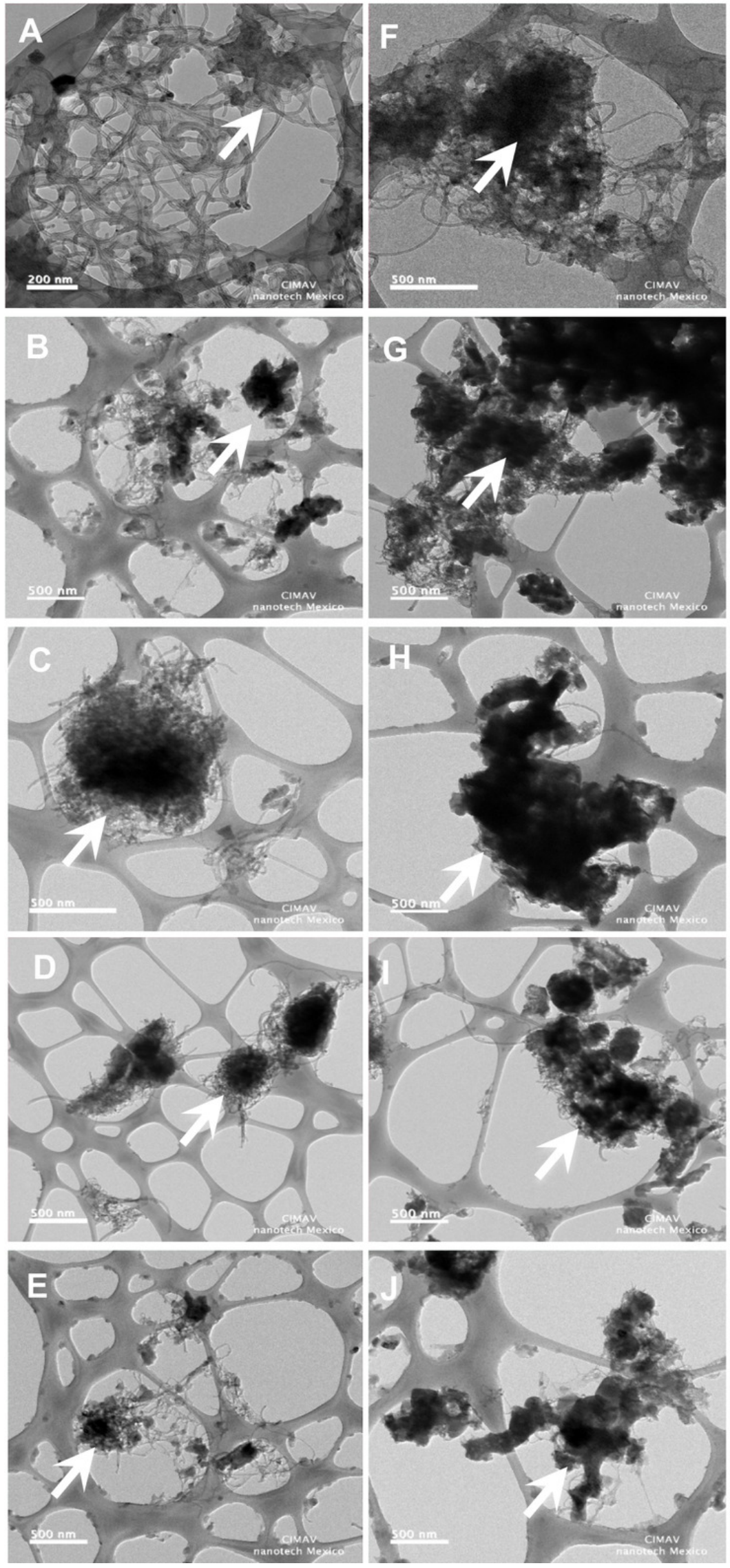
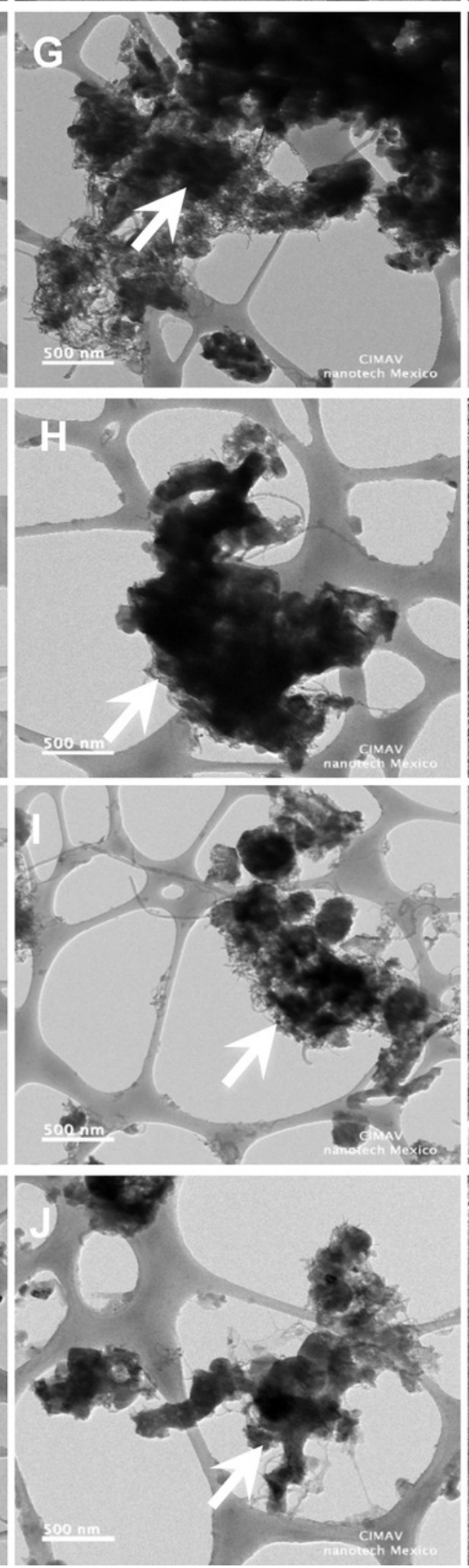

\section{Pleurotus ostreatus}
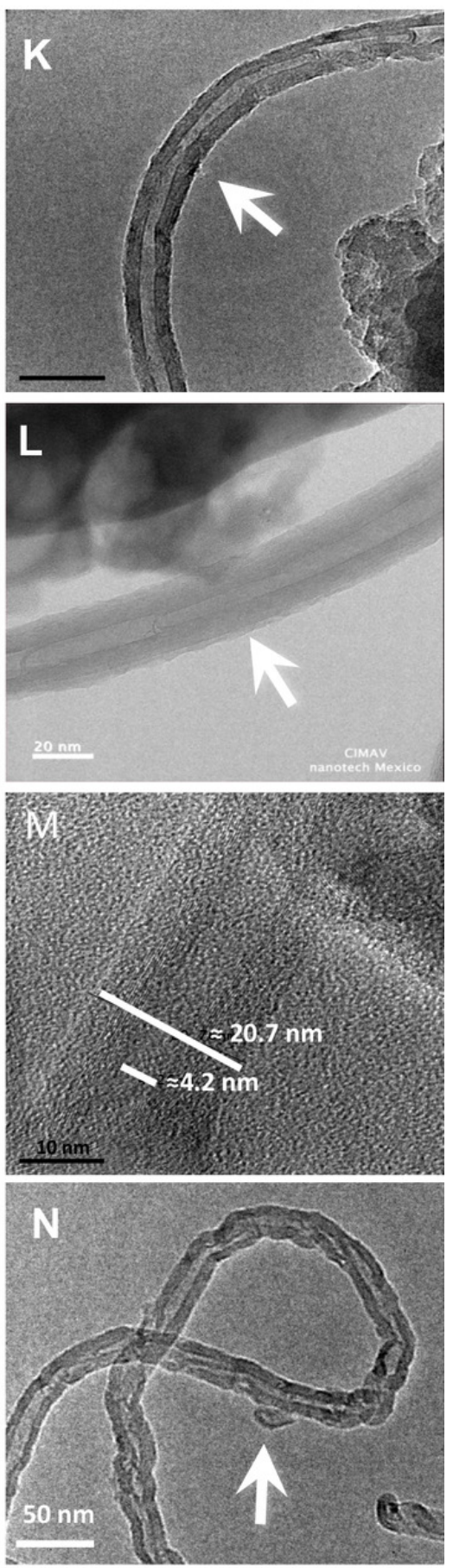

(2)

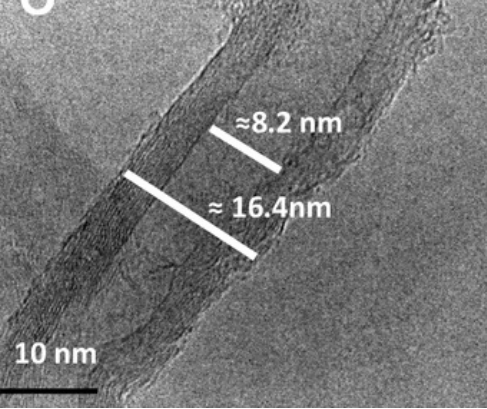




\section{Figure 6}

Mass loss on cultures of $P$. ostreatus and $P$. chrysogenum grown with MWCNTs by thermogravimetric analyses (TGA).

Samples of fugal cultures supplemented with MWCNTs were dried prior to TGA analysis. Before incubation ( 0 days) and after 28 days. P. ostreatus and $P$. chrysogenum cultures added with MWCNTs were analyzed after 28 days of cultivation. Representative plots are shown. 


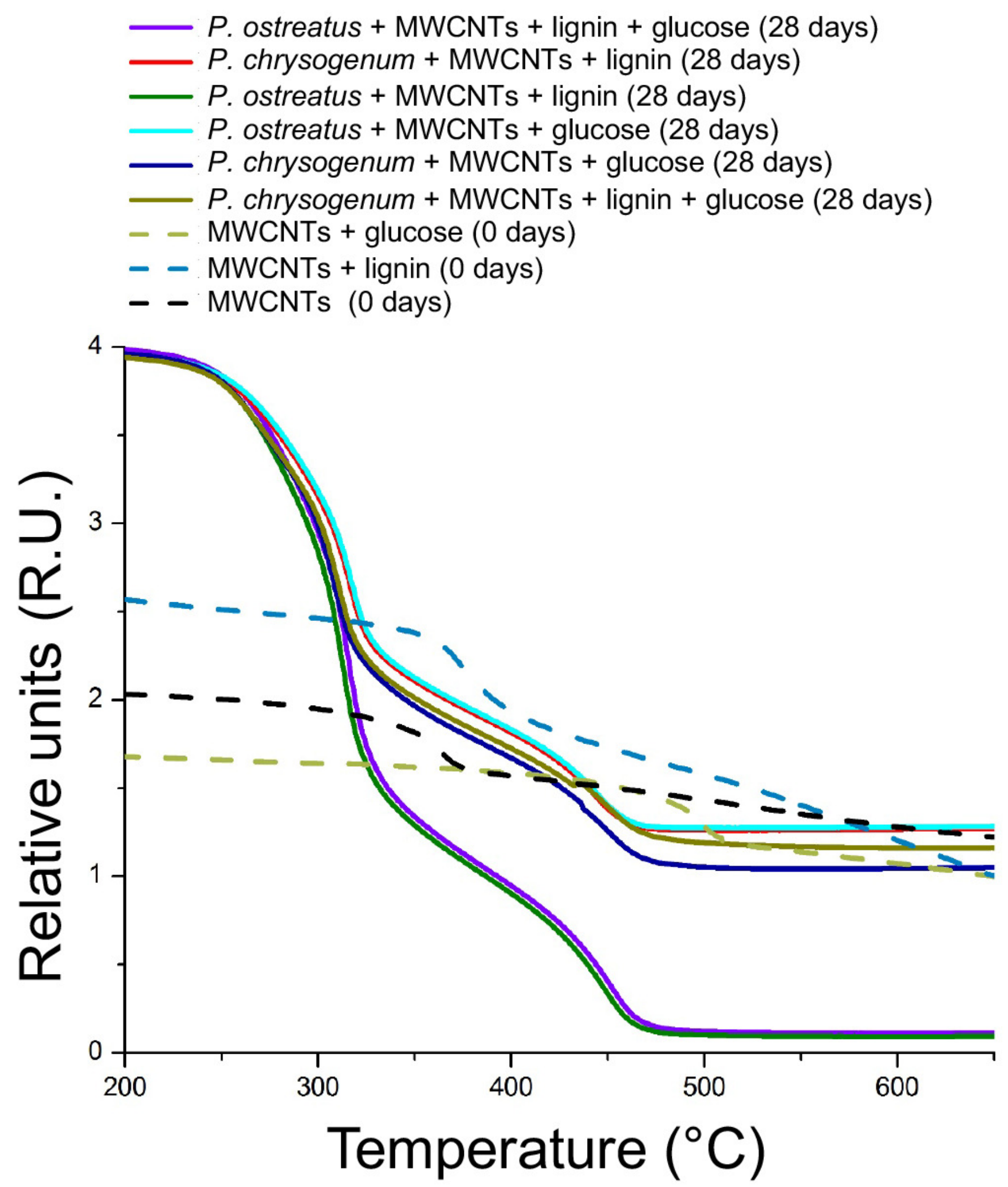




\section{Figure 7}

Toxic effects on $C$. elegans worms exposed to cultures of $P$. ostreatus and $P$. chrysogenum grown with MWCNTs.

Samples from media supplemented with MWCNTs and from fugal cultures grown by 28 days were dried and incinerated prior to worms survival test. Adult $C$. elegans worms ( 20 individuals) were incubated in $1 \mathrm{~mL}$ of culture media (control) or residues obtained after incineration at $300^{\circ} \mathrm{C}$ of samples from the $P$. ostreatus and $P$. chrysogenum fungi grown on medium with MWCNTs. Worms survival was determined at $72 \mathrm{~h}$ of exposure as described in Materials and Methods. Conditions of treatment are indicated. Bars represent the mean value \pm standard error (SE) of three independent assays with $n=20$ for each treatment. One-way ANOVA analysis was carried out, with Bonferroni post-hoc test. Values for $P<0.05$ are shown with different lower-case letters. Kinetics of worm survival are showed in Figure S2 of supplementary material. 


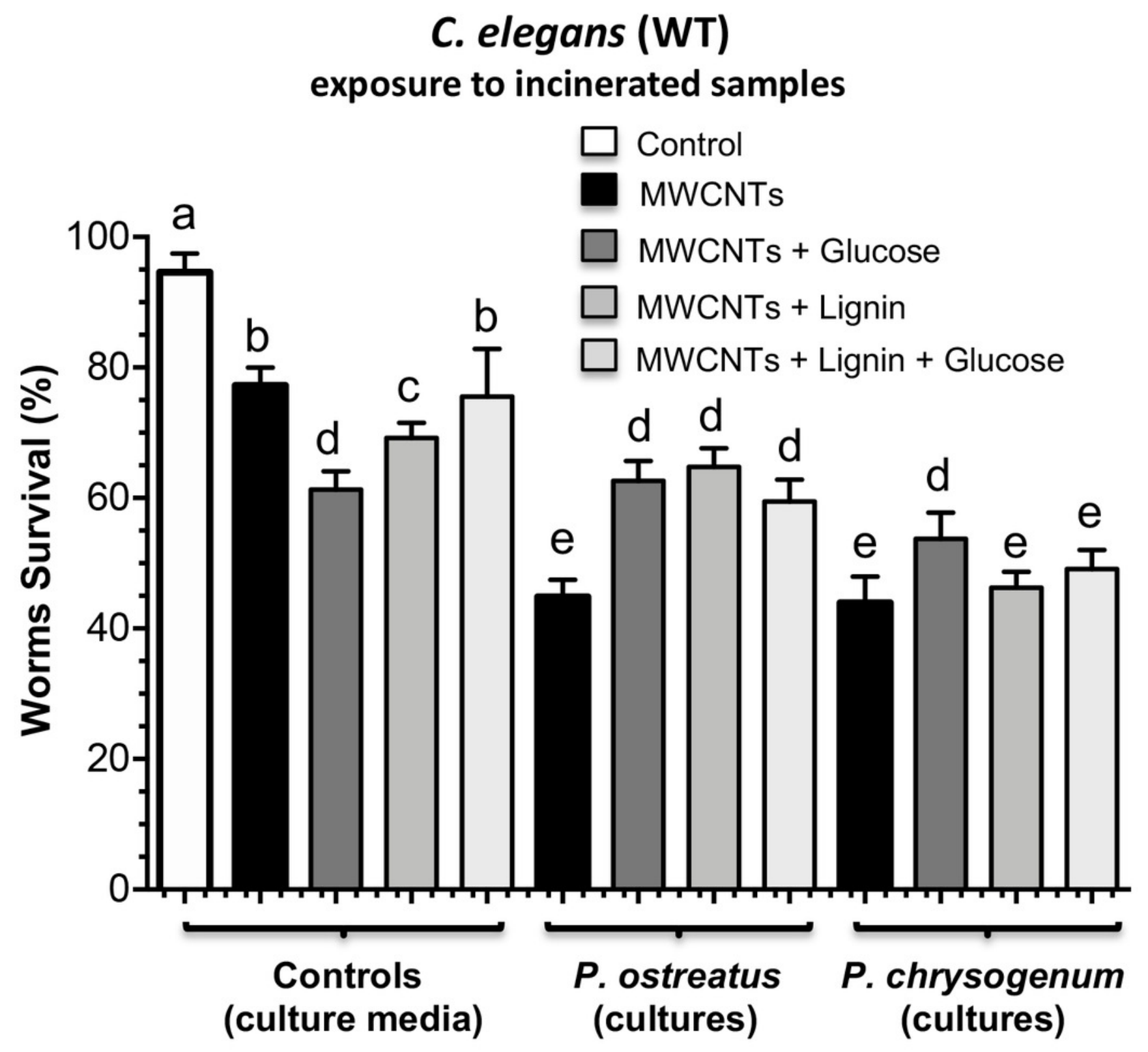




\section{Figure 8}

Microscopy fluorescence images of $C$. elegans worms treated with MWCNTs.

A) C. elegans WT treated with $10 \mu \mathrm{g} / \mathrm{mL}$ MWCNTs and superoxide generation was developed by using the Dihydroethidium fluorescent probe (DHE). (i) Worms observed with clear field at 10X magnification. (ii) Worms treated with MWCNTs by $4 \mathrm{~h}$ exposition and using DHE as probe, after observed by fluorescent field at 10X magnification. C. elegans (CL2166) GFP oxidative stress-inducible clone treated with $10 \mu \mathrm{g} / \mathrm{mL}$ MWCNTs; (iii) clear field image and (iv) fluorescent field at 10X magnification. B) C. elegans CL2166-GFP treated without or with lignin as indicated. C) C. elegans CL2166-GFP worms treated with samples taken from culture media of the $P$. ostreatus and $P$. chrysogenum fungi grown on medium with $10 \mu \mathrm{g} / \mathrm{mL}$ MWCNTs as indicated. Samples of fugal cultures were dried and incinerated prior to test. Worms observed by fluorescent field at 10X magnification. Representative images are shown. 

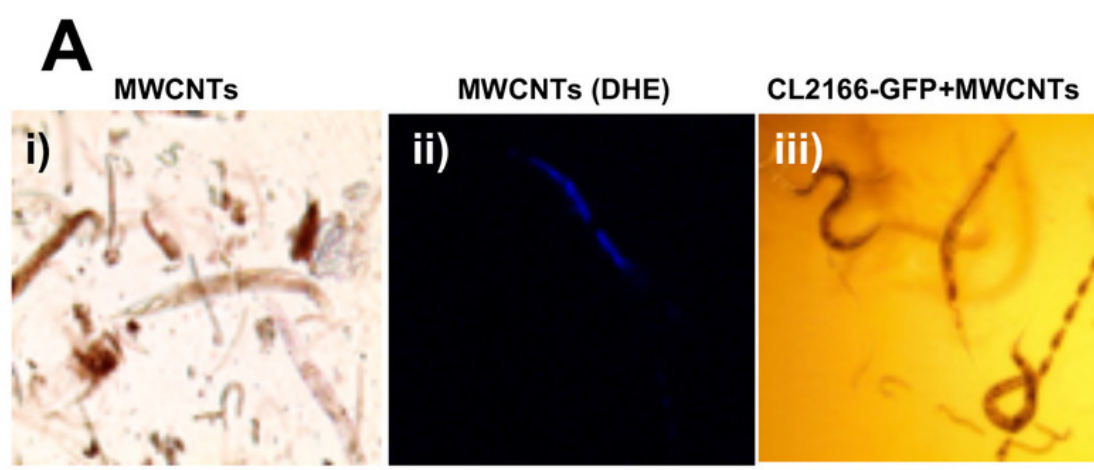

CL2166-GFP+MWCNTS

B

C. elegans (CL2166-GFP)
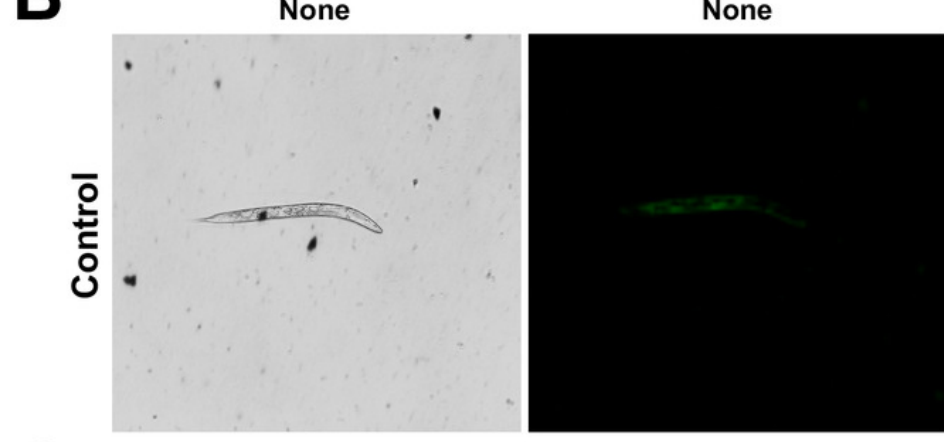

Lignin

C

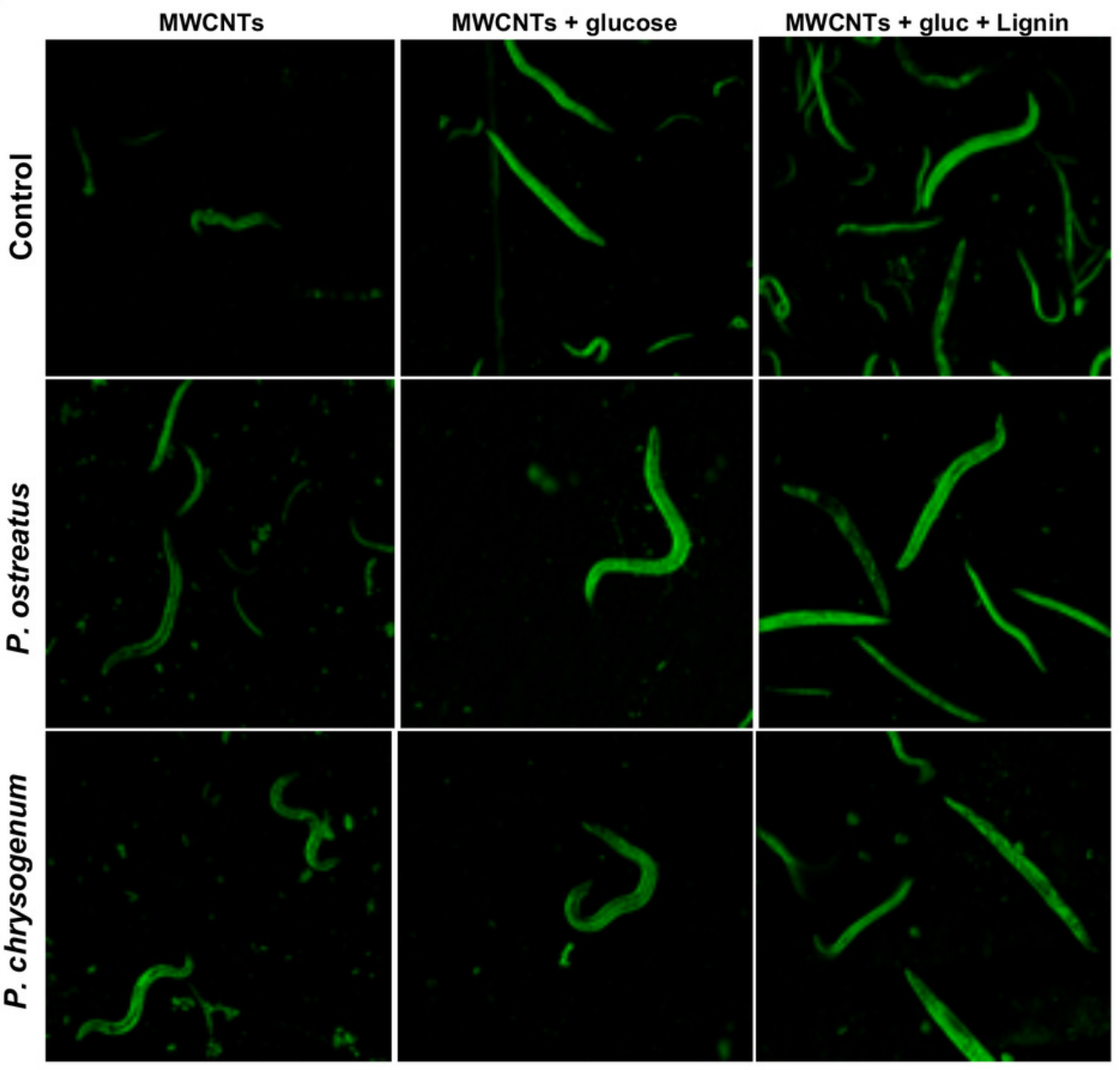

Peer] reviewing PDF | (2020:11:55553:2:0:NEW 19 Feb 2021) 


\section{Figure 9}

Reactive oxygen species production determined by fluorescence on the $C$. elegans CL2166-GFP worms treated with MWCNTs.

Worms treated by $24 \mathrm{~h}$ with the compounds at concentrations used in the culture media are indicated as control. Worms exposed to incinerated samples from fungi cultures grown on medium with MWCNTs are indicated as from $P$. ostreatus and $P$. chrysogenum. Fluorescence was determined using the Image J software in at least 10 worms of each treatment as observed in imagens showed in figure $8 \mathrm{C}$. Bars represent the mean value \pm standard error (SE), $n=10$ for each treatment. One-way ANOVA analysis was carried out, with Tukey's posthoc test. Values for $P<0.05$ are shown with different lower-case letters. 


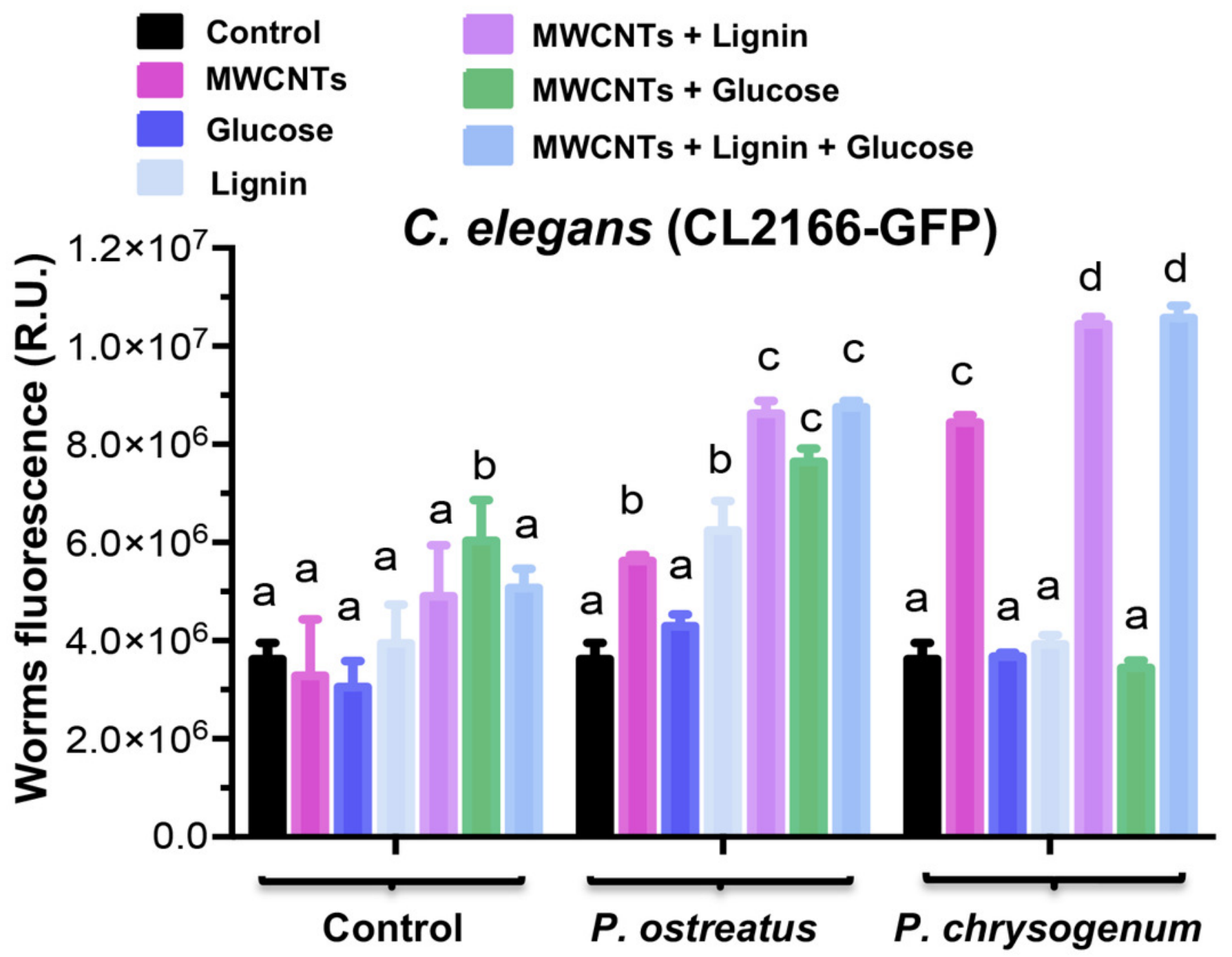

